\title{
Um estudo sobre funções de dependência e medidas de risco
}

\author{
Marcelo Gonçalves
}

TESE APRESENTADA

$\mathrm{AO}$
Instituto de Matemática e Estatística
DA
Universidade de São Paulo
PARA
OBTENÇÃO DO TÍTULO
$\mathrm{DE}$
Doutor EM CiÊNCIAS

\author{
Programa: Estatística \\ Orientador: Prof. Dr. Nikolai Kolev \\ Co-Orientador: Prof. Dr. Antonio Elias Fabris
}

Durante o desenvolvimento deste trabalho o autor recebeu auxílio financeiro da FAPESP, processo $05 / 59389-8$

São Paulo, Outubro de 2008 


\title{
Um estudo sobre funções de dependência e medidas de risco
}

\author{
Este exemplar corresponde à redação \\ final da tese devidamente corrigida \\ e defendida por Marcelo Gonçalves \\ e aprovada pela Comissão Julgadora.
}

Banca Examinadora:

- Prof. Dr. Nikolai Valtchev Kolev (orientador) - IME-USP.

- Prof. Dr. Pedro Alberto Morettin - IME-USP.

- Profa. Dra. Verônica Andrea Gonzales Lopez - UNICAMP.

- Prof. Dr. Cristiano Fernandes - PUC-RIO.

- Prof. Dr. Hélio Côrtes Vieira Lopes - PUC-RIO. 


\section{Resumo}

Começamos por estudar fronteiras para uma classe especial de medidas de risco quantis, chamadas medidas de risco distorcidas. A hipótese básica é que o conhecimento da estrutura de dependência (ou seja, da distribuição conjunta) da carteira de riscos é incompleta, fazendo com que não seja possível obter um valor exato para tais medidas. Isso é muito comum na prática. Fornecemos duas formas de obter tais limites nessa situação, apresentando seus prós e contras. A modelagem de risco, em um cenário de desconhecimento total ou parcial da distribuição conjunta dos mesmos, geralmente faz uso de cópulas. Entretanto, as cópulas vêm sendo alvo de críticas na literatura recente. Um dos motivos é que as mesmas desprezam o comportamento marginal e comprimem os dados no quadrado

unitário. Dentro desse cenário, apresentamos uma função que pode ser vista como uma alternativa e complemento ao uso de cópulas: função de dependência de Sibuya.

Palavras-chave: medidas de risco, cópulas, dependência. 


\section{Abstract}

We begin our work studying an special class of quantile risk measures, known as distorted risk measures. The basic assumption is that the risk manager does not know the complete dependence structure (that is, the risks's joint distribution) embedded in the risk's portfolio, what makes the exact computation of the risk measure an impossible task. This is a common scenario in practical problems. We present two approaches to compute bounds for the distorted risk measures in such situation, underlining the pros and cons of each one. In risk modeling, in the absence of complete knowledge regarding their joint distribution, one often relies on the copula function approach. However, copulas have been criticized in recent publications mostly because it ignores the marginal behavior and smash the data into the unity square. In order to overcome such problems we present and alternative and complement to the copula approach: the Sibuya dependence function.

Keywords: risk measures, copulas, dependence. 


\section{Sumário}

Lista de Figuras vii

$\begin{array}{ll}\text { Introdução } & 1\end{array}$

1 Fronteiras para medidas de risco quantis $\quad 3$

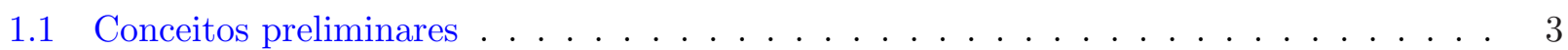

1.1.1 Medidas de risco: uma visão geral . . . . . . . . . . . . . . . . 3

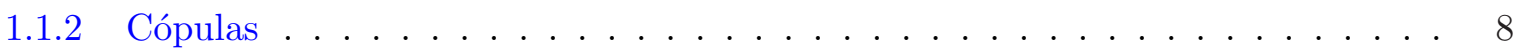

1.2 Fronteiras usando limites das distribuições acumuladas . . . . . . . . . . . . . . . . . 9

1.3 Limites via ordem estocástica . . . . . . . . . . . . . . . . . 13

1.3 .1 o caso bivariado . . . . . . . . . . . . . . . . . . 13

$1.3 .2 \quad \mathrm{O}$ caso multidimensional . . . . . . . . . . . . . . . . . . . 19

1.3.3 Aplicação aos seguros . . . . . . . . . . . . . . . . . 22

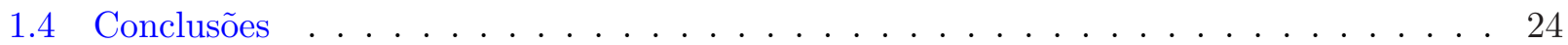

2 A função de dependência de Sibuya $\quad 25$

2.1 Algumas limitações da metodologia de cópulas . . . . . . . . . . . . . . . 25

2.2 A função de Sibuya e suas propriedades . . . . . . . . . . . . . . . . . 27

2.3 A função de dependência de Sibuya . . . . . . . . . . . . . . . . . . . . . 31 
2.3.1 Variável aleatória de Sibuya . . . . . . . . . . . . . . . . . 37

2.3.2 Limites para o valor esperado da variável aleatória de Sibuya . . . . . . . . . . 40

2.4 Distribuição de Sibuya empírica . . . . . . . . . . . . . . . . . . . . . . . . 43

2.5 A função de Sibuya em 3 dimensões . . . . . . . . . . . . . . . . . . . . . . . . 48

2.6 A função de Sibuya multidimensional . . . . . . . . . . . . . . . . . . . 57

$\begin{array}{lll}3 & \text { Conclusões } & 61\end{array}$

3.1 Considerações finais . . . . . . . . . . . . . . . . . . . . . . 61

3.2 Trabalhos futuros . . . . . . . . . . . . . . . . . . . 61

$\begin{array}{ll}\text { Referências Bibliográficas } & 65\end{array}$ 


\section{Lista de Figuras}

2.1 Curvas de Nível da função $\Lambda_{1}(x, y)=1.2,1.3,1.5 \ldots \ldots \ldots$. . . . . . . . . 32

2.2 Curvas de Nível da função $\Lambda_{2}(x, y)=1.2,1.3,1.5 \ldots \ldots$. . . . . . . . . . . 33

2.3 Diagrama de dispersão dos 14 pares (PIB, SA) observados. . . . . . . . . . . . . . 45

2.4 FDS empírica para os 14 pares $(\mathrm{PIB}, \mathrm{SA})$ observados. . . . . . . . . . . . 46 


\section{Introdução}

O presente trabalho ${ }^{1}$ tem dois objetivos. O primeiro é a busca por limites para medidas de risco quantis de uma classe de medidas de risco quantis conhecidas como 'medidas de risco distorcidas'. Quando se conhece a distribuição conjunta de riscos para a qual se deseja obter tais medidas, a tarefa é meramente um problema computacional. Todavia, o conhecimento da distribuição é uma hipótese que encontra pouca base no mundo real. O comum é que haja uma informação limitada a respeito de tal distribuição conjunta, quando não inexistente. No presente trabalho buscamos obter limites para tais medidas de risco, utilizando cópulas, sob a hipótese de conhecimento limitado a respeito da distribuição da função de risco de interesse. Nesse caso, o melhor que podemos fazer é encontrar limites, não os valores exatos das medidas.

O segundo objetivo do presente trabalho, esta relacionado com o primeiro. Trata-se da busca de uma nova função de dependência que possa, em algumas situações, substituir cópulas com vantagens. A teoria de cópulas, dentre outras limitações possíveis, ignora o comportamento marginal na especificação da estrutura de dependência entre as variáveis aleatórias. Tal fato pode ensejar uma perda de informações quando desejamos analisar o verdadeiro comportamento das variáveis envolvidas. Quando trabalhamos com medidas de risco - como no primeiro problema proposto - e desconhecemos a distribuição conjunta, geralmente usamos cópulas. Porém, como tal ferramenta negligencia o comportamento marginal, isso pode levar a um empobrecimento na análise por parte do avaliador de riscos. A função de dependência de Sibuya, aqui explorada, em muitos casos se mostra mais rica e mais complexa, que as cópulas.

A exposição que aqui se inicia esta separada em duas partes. Na primeira, buscamos obter as fronteiras para as medidas de risco, usando duas abordagens. Cada uma com seus prós e contras. Na segunda parte, exploraremos a função de dependência de Sibuya, contrapondo-a com as cópulas.

\footnotetext{
${ }^{1}$ Neste trabalho, os resultados obtidos por terceiros tem sua autoria expressa entre parênteses
} 
Estudaremos com detalhes as possíveis extensões da função de dependência de Sibuya em três dimensões, generalizaremos para o caso n-dimensional e por fim, apresentaremos um estudo sobre a dependência entre clusters usando a mesma função de dependência. 


\section{Capítulo 1}

\section{Fronteiras para medidas de risco quantis}

O cálculo de medidas de risco às vezes é muito problemático e difícil na ausência do pressuposto de independência (e.g. Darkiewicz et al., 2005), uma hipótese irrealista. Muitas vezes as marginais são conhecidas e uma informação parcial - ou até nenhuma - é conhecida sobre a forma da estrutura de dependência. Objetivamos calcular limites para medidas de risco em tal situação. Para tanto, apresentaremos duas abordagens. Uma baseada no artigo de Embrechts et al. (2003) para calcular limites para o VaR, veja também Embrechts e Puccetti (2005). A outra, será baseada no conceito de ordem estocástica. Discutiremos os prós e contras de cada método. Na seção (1.1), apresentamos uma visão geral para as medidas de risco e detalharemos as hipóteses sobre a classe de medidas de risco com que iremos trabalhar, chamadas de funções de risco 'distorcidas' - distorted risk measures. Nesta mesma seção, faremos um breve comentário sobre a teoria de cópulas. A seção (1.2) apresenta limites para as medidas de risco usando a primeira abordagem a ser introduzida. Na seção seguinte, uma segunda abordagem é utilizada para o mesmo fim. Uma conclusão sobre os resultados é feita.

\subsection{Conceitos preliminares}

Aqui, apresentaremos conceitos importantes, que serão utilizados no desenvolvimento do trabalho.

\subsubsection{Medidas de risco: uma visão geral}

Em geral uma medida de risco é definida no conjunto de riscos $\left(X_{1}, \ldots, X_{m}\right)^{1}$ tomando valores em $\Re$. As medidas de risco devem levar em conta algumas quantidades probabilísticas básicas, tais

\footnotetext{
${ }^{1}$ Risco pode ser definido como a possibilidade de ocorrência de perdas. Destarte, risco pode ser definido como uma variável aleatória $X$, que representa a perda do detentor do risco
} 
como: tendência central, variabilidade, comportamento caudal ou assimetria. No entanto, uma única medida de risco não é capaz de contemplar todas estas quantidades probabilísticas ao mesmo tempo. Deste modo, a definição de uma medida apropriada deve depender do contexto econômico. Por exemplo, considere a teoria clássica de carteiras, e.g. Markowitz (1952). A essência dessa teoria é que os investidores diversificam seus investimentos entre $n$ ativos de modo a maximizar o retorno, sujeito a uma dada exposição ao risco. Usualmente, o risco é medido pela variância do retorno da carteira.

Uma das medidas de risco mais populares no momento é o Valor em Risco (VaR). Formalmente, ele é definido como se segue, veja Jorion (1998). Para qualquer $p \in(0,1)$, o Valor-em-Risco, $\operatorname{VaR}_{p}(X)$, é o $p$-quantil da variável aleatória $X$, ou seja,

$$
\operatorname{VaR}_{p}(X)=\inf \{x \in R: F(x) \geq p\}=F^{-1}(p)
$$

onde $F(x)=P(X \leq x)$. No caso contínuo, $\operatorname{VaR}_{p}(X)$ é uma função não-decrescente e contínua à esquerda de $p$. A última fórmula implica na relação de equivalência $\operatorname{Va} R_{p}(X) \leq x \Longleftrightarrow p \leq F(x)$.

A medida $\operatorname{VaR}_{p}(X)$ tem sentido em situações onde o evento "default"” deveria ser evitado, mas a magnitude da perda devida a esse "default" não tem relevância. Para detentores de ações, o $\operatorname{VaR}_{p}(X)$ fornece uma informação útil, uma vez que evitar o "default" é uma preocupação de primeira grandeza, ao passo que o tamanho da perda associada ao "default" ocupa uma importância secundária. Entretanto, o $\operatorname{VaR}_{p}(X)$ não fornece nenhuma informação a respeito do quão pesada é a cauda direita da função distribuição.

As dificuldades em controlar e otimizar o VaR em carteiras com retornos não normais e não log-normais (veja Rockafellar e Uryasev 2000, 2002), tem levado os pesquisadores a procurar por medidas de risco baseadas em quantis. Portanto, é conveniente utilizar uma outra medida de risco, chamada de Valor em Risco Caudal (Tail Value-at-Risk) de nível $p, T V a R_{p}(X)$, e definida por

$$
T \operatorname{VaR}(X)=\frac{1}{1-p} \int_{p}^{1} \operatorname{VaR}_{q}(X) d q, \quad p \in(0,1) .
$$

Essa medida é a média aritmética dos quantis de $X$, de $p$ em diante. Note que sempre $T V a R_{p}(X)>$ $\operatorname{VaR}_{p}(X), T V a R_{0}(X)=E X$, e $T V a R_{p}(X)$ é uma função não-decrescente de $p$.

\footnotetext{
${ }^{2}$ default, em finanças, é o evento em que o devedor não cumpre suas obrigações contratuais
} 
TVaR é uma medida de risco mais adequada quando comparada ao VaR porque ela leva em conta as perdas além do nível VaR. A diferença fundamental entre VaR e TVaR como medidas de risco é: o VaR é o limite inferior "otimista" das perdas na cauda, enquanto o TVaR nos fornece o valor esperado das perdas na cauda, isto é, o TVaR pode ser interpretado como uma medida de risco conservadora.

Rockafellar e Uryasev (2000, 2002) denominam esta medida de Valor em Risco Condicional (CVaR) e fornece uma definição alternativa para o TVaR que é coerente (i.e., satisfaz os axiomas da sub-aditividade, monotonicidade, homogeneidade e invariância sobre translação; veja Artzner et al. (1999) ) mesmo no caso em que as distribuições dos retornos associados serem descontínuas. Além disso, o TVaR é caracterizado como a solução de um problema de otimização. A contribuição básica é a apresentação de uma metodologia prática de otimização do TVaR e cálculo do VaR ao mesmo tempo, o que é um modo conveniente de avaliar:

- Derivativos (opções, futuros);

- Mercado, crédito e risco operacional;

- Risco financeiro.

Hürlimann (2002) representa os limites superiores de $V_{a} R_{p}(X)$ e $T V a R_{p}(X)$ sabendo os primeiros quatro momentos de variável aleatória $X$.

Da equação (1.1) percebe-se que o TVaR utiliza somente a informação da cauda superior da distribuição. Apesar disto, esta medida não é adequada para eventos extremos, i.e., eventos de baixa freqüência e altas perdas (Wang, 2000). Wang (2000) introduziu uma medida de risco que resolve estes problemas.

\section{Medidas de Risco Distorcidas}

Considere uma função não decrescente $g:[0,1] \rightarrow[0,1]$ tal que $g(0)=0$ e $g(1)=1$, chamada função distorção. Para todo risco $X$, podemos associar uma medida de risco correspondente, através da expressão:

$$
D_{g}(X)=\int_{-\infty}^{0}(1-g(1-F(t))) d t+\int_{0}^{\infty} g(1-F(t)) d t
$$


Se $X$ for não negativo, então:

$$
D_{g}(X)=\int_{0}^{\infty} g(1-F(t)) d t=\int_{0}^{1} F^{-1}(1-q) d g(q) .
$$

A ultima relação à esquerda só é válida se $g($.$) for crescente. No que se segue, vamos por simplicidade$ assumir que os riscos são não negativos (como no caso de um seguro, em que $X$ representa a perda paga pela seguradora, em valor absoluto).

Em relação à (1.2), $\operatorname{VaR}_{p}(X), T V a R_{p}(X)$ e a transformação de Wang $W T_{p}(X)$ (usada para definir uma medida de risco adequada para eventos de alta frequência e baixas perdas, veja Wang, 2000) discutidas acima, correspondem às funções distorções, $g(x)=I(x>1-p)$, onde $I($.) denota a função indicadora, $g(x)=\min \left(\frac{x}{1-p}, 1\right)$ e $g(x)=\Phi\left[\Phi^{-1}(x)+\Phi^{-1}(p)\right]$ em que $\Phi($.$) é a função$ distribuição normal padrão, respectivamente.

Varias outras funções podem ser ecolhidas para $g$, a maior parte discutidas em Wang (1996). Para cada família, o parâmetro $p$ está no intervalo $(0,1)$.

- Transformação de potência:

$$
g_{p}(x)=x^{p}
$$

- Transformação de potência dual:

$$
g_{p}(x)=1-(1-x)^{\frac{1}{p}}
$$

- Transformação de Dennensberg:

$$
g_{p}(x)= \begin{cases}(1+p) x & \text { for } 0 \leq x \leq \frac{1}{2} \\ p+(1-p) x & \text { for } \frac{1}{2} \leq x \leq 1\end{cases}
$$

- Transformação de Gini:

$$
g_{p}(x)=(1+p) x-p x^{2} ;
$$

- Transformação da raiz quadrada:

$$
g_{p}(x)=\frac{\sqrt{1-\ln (p) x}-1}{\sqrt{1-\ln (p)}-1}
$$


- Transformação exponencial:

$$
g_{p}(x)=\frac{1-p^{x}}{1-p}
$$

- Transformação logarítmica:

$$
g_{p}(x)=\frac{\ln (1-\ln (p) x)}{\ln (1-\ln (p))}
$$

Nosso intuito é encontrar fronteiras para as medidas de risco definidas por (1.2), todas elas baseadas em quantis. Tais funções apresentam uma série de propriedades desejáveis do ponto de vista da teoria econômica; veja Yaari (1987)

Passemos, agora, a analisar situações gerais em que se demanda tais medidas de risco.

Considere um tomador de decisões frente a um certo número de riscos, i.e., perdas futuras aleatórias. No que segue, sempre consideraremos perdas como variáveis aleatórias, i.e., pagamentos aleatórios que devem ser feitos. Para um dado conjunto de riscos $X_{1}, \ldots, X_{m}$, definimos $\psi: \Re^{m} \rightarrow \Re$ como uma função do conjunto de variáveis aleatórias representando os riscos. Usualmente se está interessado em calcular certas grandezas de $\psi\left(X_{1}, \ldots, X_{m}\right)$, como momentos ou quantis. Exemplos típicos de $\psi($.$) incluem:$

1. $\psi_{1}\left(x_{1}, \ldots, x_{m}\right)=x_{1}+\ldots+x_{m}$. Em um contexto de seguros, freqüentemente se está interessado na função distribuição da soma de variáveis aleatórias. Tal soma aparece quando se consideram os sinistros agregados de uma carteira de seguros em um dado período de referência. Também a soma ocorre quando se consideram pagamentos descontados relacionados a uma única apólice ou a uma carteira, em diferentes pontos futuros do tempo. No caso da função quantil, o objeto de interesse seria o Valor-em-Risco da posição conjunta $X_{1}+\ldots+X_{m}$;

2. $\psi_{2}\left(x_{1}, \ldots, x_{m}\right)=\sum_{i=1}^{m}\left(x_{i}-k\right)^{+}$, onde $a^{+}=\max (a, 0), k>0$. Este caso corresponde à expressão funcional de um acordo de resseguro do excesso de perda além de $k$. Os $X_{i}$ 's poderiam ser sinistros individuais ou perdas de resseguro devido a diferentes ramos de negócios. A esperança $E\left(\psi_{2}().\right)$ está relacionada com prêmios de seguros;

3. $\psi_{3}\left(x_{1}, \ldots, x_{m}\right)=\left(\sum_{i=1}^{m} x_{i}-k\right)^{+}$. Esta forma tem uma interpretação em derivativos (e.g. opções asiáticas) ou resseguro do tipo "stop-loss".

Na literatura de finanças e atuária podem ser encontradas muitas formas analíticas para medidas de risco de $\psi($.$) , correspondendo a opções exóticas, derivativos de crédito e de cestas de ativos,$ 
cobertura de riscos operacionais; veja Embrechts et al. (2003), Patton (2003), Georges et al. (2001), entre outros.

$\mathrm{Na}$ maioria dos casos primeiro se trabalha com as marginais e então se introduz a estrutura de dependência via a escolha de uma cópula. Uma vez que a estrutura de dependência é especificada, o cálculo das quantidades dependentes da função distribuição de $\psi\left(X_{1}, \ldots, X_{m}\right)$ se torna uma questão puramente computacional.

Infelizmente, em muitas situações somente uma informações parcial ou nenhuma informação sobre a cópula correspondente a $\left(X_{1}, \ldots, X_{m}\right)$ é conhecida. Nestes casos é útil determinar os limites da função distribuição de $\psi\left(X_{1}, \ldots, X_{m}\right)$.

Tais limites no caso em que $m=2$ e $\psi\left(x_{1}, x_{2}\right)=x_{1}+x_{2}$ e a estrutura de dependência (cópula) não é especificada, são dados por Makarov (1981) e Frank et al. (1987) como segue. Seja $C_{L}(u, v)=$ $\max (u+v-1,0)$ e $z \in \Re$. Então,

$$
P(X+Y \leq z) \geq \sup _{x+y=z} C_{L}(F(x), G(y))=\phi(z)
$$

onde $\phi(z)=\psi(x, y)$ calculado em $x, y$ como se segue

$$
\sup _{x+y=z} C_{L}(F(x), G(y))=\psi(x, y)
$$

Por fim,

$$
\psi^{-1}(p)=\inf _{C_{L}(u, v)=p}\left\{F^{-1}(u)+G^{-1}(v)\right\}, p \in(0,1)
$$

e $\operatorname{VaR}_{p}(X+Y) \leq \psi^{-1}(p)$. Denuit et al. (1999) estenderam esse resultados para o caso onde $m \geq 3$, quando as marginais são as mesmas.

\subsubsection{Cópulas}

A dependência entre as variáveis aleatórias $X_{1}, \ldots, X_{m}$ é completamente descrita pela função de distribuição conjunta $H\left(x_{1}, \ldots, x_{m}\right)$. $H($.$) é uma função distribuição conjunta com marginais$ $F_{X_{1}}\left(x_{1}\right), \ldots, F_{X_{m}}\left(x_{m}\right)$. Suponha que se transforme o vetor aleatório $\left(X_{1}, \ldots, X_{m}\right)$ componente a componente via $F_{X_{i}}\left(X_{i}\right)=U_{i}, i=1, \ldots, m$, para que ele tenha distribuições marginais uniformes $U(0,1)$. Deste modo, o teorema de Sklar, veja Nelsen (1999), afirma que existe uma função $C($.$) ,$ 
denominada cópula $n$-dimensional tal que

$$
H\left(x_{1}, \ldots, x_{m}\right)=C\left(F_{X_{1}}\left(x_{1}\right), \ldots, F_{X_{m}}\left(x_{m}\right)\right)
$$

Se $F_{X_{1}}, \ldots, F_{X_{m}}$ são todas contínuas, então $C($.$) é única, caso contrário C($.$) é unicamente deter-$ minada no conjunto $\operatorname{Im}\left(F_{X_{1}}\right) \times \ldots \times \operatorname{Im}\left(F_{X_{m}}\right)$, onde $\operatorname{Im}($.$) representa a imagem da função. Da$ expressão (1.3) temos que a cópula $C($.) é uma função que relaciona as funções distribuição marginais com a função distribuição conjunta.

Formalmente, cópula é uma função distribuição conjunta

$$
C\left(u_{1}, \ldots, u_{m}\right)=P\left(U_{1} \leq u_{1}, \ldots, U_{m} \leq u_{m}\right), \quad 0 \leq u_{i} \leq 1
$$

cujas marginais $U_{i} \sim U(0,1), i=1, \ldots, m$.

A recíproca da relação (1.3) é a seguinte: Seja $H($.$) uma função distribuição conjunta com$ marginais $F_{X_{1}}\left(x_{1}\right), \ldots, F_{X_{m}}\left(x_{m}\right)$ contínuas e $F_{X_{1}}^{-1}, \ldots, F_{X_{m}}^{-1}$ suas inversas, então para qualquer $u_{i} \in$ $[0,1]$ existe uma cópula $m$-dimensional $C($.$) tal que$

$$
C\left(u_{1}, \ldots, u_{m}\right)=H\left(F_{X_{1}}^{-1}\left(u_{1}\right), \ldots, F_{X_{m}}^{-1}\left(u_{m}\right)\right)
$$

A relação (1.3) implica que podemos acoplar distribuições univariadas, de qualquer tipo, com qualquer cópula, e teremos definido uma distribuição multivariada válida. Este resultado é útil porque usualmente temos alguma idéia sobre as distribuições univariadas, mas nossa intuição raramente indica a escolha de distribuições multivariadas disponíveis. Outro fato importante que pode ser extraído da relação (1.4) é que podemos estudar o fenômeno de dependência entre as variáveis sem fixar as distribuições marginais; veja Nelsen (2006).

No capítulo 2, apresentaremos uma abordagem crítica a teoria das cópulas.

Passemos, agora, a primeira abordagem para se obter as fronteiras para os riscos quantis.

\subsection{Fronteiras usando limites das distribuições acumuladas}

Sejam $\left(X_{1}, \ldots, X_{m}\right)$ variáveis aleatórias com distribuições marginais $F_{X_{1}}, \ldots, F_{X_{m}}$ e cópula $C($.$) .$ Seja $\psi: \Re^{m} \rightarrow \Re$ uma função crescente e contínua à direita no último argumento. Denote por $\psi_{k}$ a função $\psi$ com argumentos fixos $x_{i_{1}}, \ldots, x_{i_{k}}$ para $1 \leq i_{1}<\ldots<i_{k} \leq m$. Considere a cópula dual de 
$C($.$) dada por,$

$$
C^{d}\left(u_{1}, \ldots, u_{m}\right)=P\left(\left\{U_{1} \leq u_{1}\right\} \cup \cdots \cup\left\{U_{m} \leq u_{m}\right\}\right) .
$$

Por exemplo, para $m=2$ tem-se $C^{d}\left(u_{1}, u_{2}\right)=u_{1}+u_{2}-C\left(u_{1}, u_{2}\right)$.

Defina,

$$
\begin{gathered}
\tau_{C, \psi}(s)=\sup _{x_{1}, \ldots, x_{m-1} \in \Re} C\left(F_{X_{1}}\left(x_{1}\right), \ldots, F_{X_{m-1}}\left(x_{m-1}\right), F_{X_{m}}\left(\psi_{m-1}^{-1}(s)\right)\right), \\
\sigma_{C, \psi}(s)=\int_{\psi \leq s} d C\left(F_{X_{1}}\left(x_{1}\right), \ldots, F_{X_{m}}\left(x_{m}\right)\right), \\
\rho_{C, \psi}(s)=\inf _{x_{1}, \ldots, x_{m-1} \in \Re} C^{d}\left(F_{X_{1}}\left(x_{1}\right), \ldots, F_{X_{m-1}}\left(x_{m-1}\right), F_{X_{m}}\left(\psi_{m-1}^{-1}(s)\right)\right),
\end{gathered}
$$

em que $\psi_{m-1}^{-1}(s)=\sup \left\{x \in \Re \mid \psi_{m-1}(x) \leq s\right\}$ é a função inversa generalizada contínua à direita, de $\psi_{m-1}(s)$.

A seguinte afirmação desempenha um papel crucial em nossas considerações.

Teorema 1 (Embrechts et al, 2003). Sob as notações acima, se uma cópula $C($.$) associada a$ $\left(X_{1}, \ldots, X_{m}\right)$ satisfaz $C \geq C_{0}$ e $C^{d} \leq C_{1}^{d}$ para algum par de cópulas $C_{0} e C_{1}$, ponto a ponto, então

$$
\tau_{C_{0}, \psi}(s) \leq \sigma_{C, \psi}(s) \leq \rho_{C_{1}, \psi}(s)
$$

Observe que $\sigma_{C, \psi}(s)=P\left(\psi\left(X_{1}, \ldots, X_{m}\right) \leq s\right)=G_{\psi}(s)$, onde $G_{\psi}(s)$ denota a distribuição acumulada de $\psi\left(x_{1}, \ldots, x_{m}\right)$. Tem-se também que, $\tau_{C_{0}, \psi}(s)$ e $\rho_{C_{1}, \psi}(s)$ são funções distribuição, i.e., a desigualdade (1.8) fornece fronteiras para a função $\psi$ de riscos dependentes $X_{1}, \ldots, X_{m}$. Como não existe necessariamente uma única cópula satisfazendo (1.8), assume-se, adicionalmente, que existe uma cópula $C\left(\right.$.) tal que $C \geq C_{0}$ e $C^{d} \leq C_{1}^{d}$. As cópulas $C_{0}$ e $C_{1}$ representam a informação parcial disponível sob a estrutura de dependência de $\left(X_{1}, \ldots, X_{m}\right)$. Em Embrechts et al. (2003) é proposta uma metodologia para se encontrar fronteiras para $\operatorname{VaR}\left(G_{\psi}(s)\right)$.

$\mathrm{Na}$ maioria dos casos, as fronteiras em (1.8) não têm forma fechada. Em geral, tem-se que fazer uso de aproximações numéricas como proposto em Williamson and Downs (1990). O algoritmo deles é baseado na discretização de $\tau_{C_{0}, \psi}(s), \rho_{C_{1}, \psi}(s)$ e no princípio da dualidade, veja Frank et al. (1987). 
Explicaremos, agora, como usar o Teorema 1 para obter os resultados desejados.

A expressão (1.8) nos permite obter as fronteiras utilizando-se a seguinte expressão:

$$
\int_{0}^{\infty} g\left(1-\rho_{C_{1}, \psi}(t)\right) d t \leq D_{g}(X) \leq \int_{0}^{\infty} g\left(1-\tau_{C_{0}, \psi}(t)\right) d t
$$

Por exemplo, sejam $T V a R_{p}(\psi), P T_{p}(\psi)$ e $G T_{p}(\psi)$ as notações para o $\operatorname{TVaR}\left(g(x)=\min \left(1, \frac{x}{1-p}\right)\right)$, a Transformação de Potência $\left(g(x)=x^{p}\right)$ e a Transformação de Gini $\left(g(x)=(1+p) x-p x^{2}\right)$, cujas medias de risco são definidas por (1.2), utilizando-se as funções de distorção correspondentes, respectivamente. A transformação de Potência incorpora a aversão ao risco relacionada a incerteza: quanto menor o valor de $p$, maior a aversão. Para a Transformação de Gini, o cálculo do prêmio se baseia no desvio absoluto em relação a mediana. Denneberg (1990) generaliza o princípio de Gini.

Aplicando (1.8), obtemos os seguintes limites:

$$
\begin{gathered}
\frac{1}{1-p} \int_{p}^{1} \rho_{C_{1}, \psi}^{-1}(q) d q \leq T \operatorname{Ta} R_{p}(\psi) \leq \frac{1}{1-p} \int_{p}^{1} \tau_{C_{0}, \psi}^{-1}(q) d q \\
\int_{0}^{\infty}\left[1-\rho_{C_{1}, \psi}(t)\right]^{p} d t \leq P T_{p}(\psi) \leq \int_{0}^{\infty}\left[1-\tau_{C_{0}, \psi}(t)\right]^{p} d t
\end{gathered}
$$

$\mathrm{e}$

$$
\begin{gathered}
\int_{0}^{\infty}\left\{(1+p)\left[1-\rho_{C_{1}, \psi}(t)\right]-p\left[1-\rho_{C_{1}, \psi}(t]^{2}\right\} d t \leq G T_{p}(\psi) \leq\right. \\
\int_{0}^{\infty}\left\{(1+p)\left[1-\tau_{C_{0}, \psi}(t)\right]-p\left[1-\tau_{C_{0}, \psi}(t)\right]^{2}\right\} d t
\end{gathered}
$$

Veja Goncalves et al. (2005).

Exemplo 1. Sejam $X_{1}$ e $X_{2}$ dois riscos quaisquer com distribuições uniformes em [0,1], e $\psi\left(X_{1}, X_{2}\right)=$ $X_{1}+X_{2}$. Na ausência de qualquer informação, sempre podemos usar que $C_{0}$ é o limite inferior de Fréchet e que $C_{1}^{d}=C_{0}^{d}=\min \left\{x_{1}+x_{2}, 1\right\}$. Nesse caso, as desigualdades supracitadas se tornam

$$
\frac{1+p}{2} \leq T \operatorname{Va} R_{p}\left(X_{1}+X_{2}\right) \leq \frac{3+p}{2},
$$




$$
\frac{1}{p+1} \leq P T_{p}\left(X_{1}+X_{2}\right) \leq \frac{p+2}{p+1}
$$

$\mathrm{e}$

$$
\frac{3+p}{6} \leq G T_{p}\left(X_{1}+X_{2}\right) \leq \frac{9+p}{6}
$$

Como ja foi dito anteriormente, em muitos casos devemos fazer uso de métodos numéricos para calcular esses limites. Usando da metodologia de Williamson e Downs (1990) (para calcular as inversas $\rho_{C_{1}, \psi}^{-1}(q)$ e $\tau_{C_{0}, \psi}^{-1}(q)$ ), podemos proceder da seguinte forma para obtermos limites numéricos para a medida de risco, quando a função de distorção $g($.$) for crescente e os riscos forem não-negativos.$

Expressemos (1.2) como se segue.

$$
D_{g}(X)=-\int_{0}^{1} F^{-1}(u) d g(1-u)
$$

Agora, defina $p_{i}=\frac{i}{N}$, com $i=1,2, \ldots, N-1$, onde $N$ denota o número de pontos que escolhemos para dividir o intervalo $[0,1]$. Então, utilizando (1.8) e (1.10), obtemos o seguinte limite para $D_{g}(\psi)$

$$
D_{g}(\psi) \leq-\sum_{i=0}^{N-1} \rho_{C_{1}, \psi}^{-1}\left(p_{i+1}\right) \int_{p_{i}}^{p_{i+1}} d g(1-u)=\sum_{i=1}^{N-1} \rho_{C_{1}, \psi}^{-1}\left(p_{i+1}\right)\left[g\left(1-p_{i}\right)-g\left(1-p_{i+1}\right)\right]
$$

Analogamente, o limite inferior segue.

$$
D_{g}(\psi) \geq-\sum_{i=0}^{N-1} \tau_{C_{0}, \psi}^{-1}\left(p_{i}\right) \int_{p_{i}}^{p_{i+1}} d g(1-u)=\sum_{i=0}^{N-1} \tau_{C_{0}, \psi}^{-1}\left(p_{i}\right)\left[g\left(1-p_{i}\right)-g\left(1-p_{i+1}\right)\right]
$$

Resumindo, se a função de distorção $g($.) for crescente podemos calcular numericamente os limites para $D_{g}(\psi)$ usando as desigualdades

$$
\sum_{i=0}^{N-1} \tau_{C_{0}, \psi}^{-1}\left(p_{i}\right)\left[g\left(1-p_{i}\right)-g\left(1-p_{i+1}\right)\right] \leq D_{g}(\psi) \leq \sum_{i=0}^{N-1} \rho_{C_{1}, \psi}^{-1}\left(p_{i+1}\right)\left[g\left(1-p_{i}\right)-g\left(1-p_{i+1}\right)\right]
$$


Infelizmente, não há garantias que os limites obtidos nessa seção serão precisos ('sharp') ${ }^{3}$. O motivo é que, teoricamente, para cada argumento "s" dado em (1.8), sabemos a cópula $C($.$) que$ atinge o limite superior (inferior). A integração é feita sob todo um conjunto de "s" para a mesma cópula $C($.$) e como consequência, não há garantia de que esses limites serão 'sharp'. Na verdade,$ mais para frente, veremos que esses limites falham em ser 'sharp'.

Na próxima seção veremos como obter os melhores limites possíveis em algumas situações.

\subsection{Limites via ordem estocástica}

Nesta seção obteremos limites para as medidas de risco quantis apresentadas usando ordem estocástica. Inicialmente, nos focaremos no caso bivariado.

\subsubsection{O caso bivariado}

Aqui, encontraremos limites para funções de dois riscos. É preciso, de início, apresentar vários conceitos relacionados a ordem estocástica (veja Müller and Stoyan, 2002).

- Uma função $f: \Re^{2} \rightarrow \Re$ é dita supermodular se

$$
f\left(x_{1}+\epsilon, x_{2}+\delta\right)+f\left(x_{1}, x_{2}\right) \geq f\left(x_{1}, x_{2}+\delta\right)+f\left(x_{1}+\epsilon, x_{2}\right)
$$

para todo $x \in \Re^{2}$ e todo $\epsilon, \delta>0$;

- Considere os vetores aleatórios $\mathbf{X}=\left(X_{1}, X_{2}\right)$ e $\mathbf{Y}=\left(Y_{1}, Y_{2}\right)$. Dizemos que $\mathbf{X}$ é menor que $\mathbf{Y}$ na ordem supermodular (escrito como $\mathbf{X} \leq_{s m} \mathbf{Y}$ ) se

$$
E f(\mathbf{X}) \leq E f(\mathbf{Y})
$$

para toda função supermodular $f$ tal que a esperança exista ;

- Sejam X e Y duas variáveis aleatórias. Dizemos que X é menor que Y na ordem 'stop-loss' (denotado por $X \leq_{s l} Y$ ) se

$$
\operatorname{Eh}(X) \leq E h(Y)
$$

para toda função convexa crescente $h$ tal que a esperança exista.

\footnotetext{
${ }^{3}$ precisos, ou 'sharp', siginifica que existe uma cópula $C_{a}($.$) tal que o limite inferior da desigualdade é atingido, e$ uma cópula $C_{b}($.$) que atinge o limite superior.$
} 
Os próximos dois teoremas são de Müller (1997). O primeiro, fornece uma relação entre ordem supermodular e ordem 'stop-loss' (adaptado ao caso bivariado) e o segundo nos dá uma relação de equivalência na ordem supermodular :

Teorema 2 (Müller, 1997). Sejam $\mathbf{X}$ e $\mathbf{Y}$ dois vetores aleatórios bivariados tais que $\mathbf{X} \leq_{\text {sm }} \mathbf{Y}$. Defina $S=X_{1}+X_{2}$ e $S^{\prime}=Y_{1}+Y_{2}$, Então $S \leq_{s l} S^{\prime}$.

Teorema 3 (Müller, 1997). Sejam $\mathbf{X}=\left(X_{1}, X_{2}\right)$ e $\mathbf{Y}=\left(Y_{1}, Y_{2}\right)$ dois vetores aleatórios com marginais iguais. Então, as seguintes relações são equivalentes :

- $\mathbf{X} \leq_{s m} \mathbf{Y}$;

- $P\left(X_{1}<s, X_{2}<t\right) \leq P\left(Y_{1}<s, Y_{2}<t\right)$ para todo $s, t \in \Re$;

- $P\left(X_{1}>s, X_{2}>t\right) \leq P\left(Y_{1}>s, Y_{2}>t\right)$ para todo $s, t \in \Re$.

Observação. A segunda relação do Teorema 3 serve como definição de ordem ortante superior - 'superior orthant order' enquanto a terceira é conhecida como ordem ortante inferior - inferior orthant order.

A próxima afirmação nos fornece uma relação entre função de distorção côncava e a ordem 'stoploss'. Ela desempenha um papel fundamental para que possamos obter os resultados desejados.

Teorema 4 (Wang et al., 1997). Se a função de distorção g é côncava, então se $X \leq_{\text {sl }} Y$, segue que $D_{g}(X) \leq D_{g}(Y)$.

Nas hipóteses do Teorema 3, é verdade a seguinte Proposição:

Proposição 1. Se uma cópula $C$ satisfaz $C_{0} \leq C \leq C_{1}$, para todo $(u, v) \in[0,1]^{2}$ e um par de cópulas $C_{0}$ e $C_{1}$, então, para toda função de distorção côncava $g$,

$$
D_{g}\left(Z_{1}+Z_{2}\right) \leq D_{g}\left(X_{1}+X_{2}\right) \leq D_{g}\left(Y_{1}+Y_{2}\right),
$$


onde $D_{g}$ é dada por (1.2) e os vetores $\mathbf{X}=\left(X_{1}, X_{2}\right), \mathbf{Z}=\left(Z_{1}, Z_{2}\right)$ e $\mathbf{Y}=\left(Y_{1}, Y_{2}\right)$ são representados pelas cópulas $C, C_{0}$ e $C_{1}$, respectivamente, sendo a marginal de $X_{1}$ igual a de $X_{2}$, de $Y_{1}$ igual a de $Y_{2}$ e a de $Z_{1}$ igual a de $Z_{2}$. Além disso, os limites são 'sharp'.

Prova. A desigualdade $C \leq C_{1}$ para todo $s$ e $t$ e a igualdade das marginais supramencionadas implica que

$$
P\left(X_{1}<s, X_{2}<t\right) \leq P\left(Y_{1}<s, Y_{2}<t\right)
$$

Pelo Teorema 3, segue-se $\mathbf{X} \leq_{s m} \mathbf{Y}$. Já o Teorema 2 nos fornece

$$
X_{1}+X_{2} \leq_{s l} Y_{1}+Y_{2}
$$

Finalmente, aplicando-se o Teorema 4, obtemos a seguinte relação

$$
D_{g}\left(X_{1}+X_{2}\right) \leq D_{g}\left(Y_{1}+Y_{2}\right)
$$

Para provar que $D_{g}\left(X_{1}+X_{2}\right) \geq D_{g}\left(Z_{1}+Z_{2}\right)$, procedemos analogamente, primeiro mostrando que $Z \leq_{s m} X$. Para verificar que os limites são 'sharp', considere os casos $\mathbf{X}=\mathbf{Y}$ q.c e $\mathbf{X}=\mathbf{Z}$ q.c.

O próximo Teorema afirma que funções crescentes aplicadas nos componentes $X_{i}$ de $\mathbf{X}$ e $Y_{i}$ de $\mathbf{Y}$, para $i=1, \ldots, n$, preserva a ordem supermodular.

Teorema 5 (Müller, 1997). Assuma que $\mathbf{X} \leq_{\text {sm }} \mathbf{Y}$ e seja $\phi_{1}, \ldots, \phi_{n}$ funções crescentes no suporte de $\left(X_{i}, Y_{i}\right)$, para $i=1,2, \ldots, n$. Então,

$$
\left(\phi_{1}\left(X_{1}\right), \ldots, \phi_{n}\left(X_{n}\right)\right) \leq_{s m}\left(\phi_{1}\left(Y_{1}\right), \ldots, \phi_{n}\left(X_{n}\right)\right)
$$

Devemos notar que o Teorema 5 nos dá uma relação entre a ordem 'stop-loss' e a soma dos componentes de um vetor aleatório. Podemos alcançar um resultado mais amplo, utilizando esse teorema (nas hipóteses do Teorema 3), como mostra a seguinte Proposição. 
Proposição 2. Sejam $\phi_{1}$ e $\phi_{2}$ funções crescentes no suporte de $X_{1}$ e $X_{2}$ respectivamente. Se uma cópula $C$ satisfaz $C_{0} \leq C \leq C_{1}$, para todo $(u, v) \in[0,1]^{2}$, e pares de cópulas $C_{0}$ e $C_{1}$, então, sob as mesmas condições da Proposição 1,

$$
D_{g}\left(\phi_{1}\left(Z_{1}\right)+\phi_{2}\left(Z_{2}\right)\right) \leq D_{g}\left(\phi_{1}\left(X_{1}\right)+\phi_{2}\left(X_{2}\right)\right) \leq D_{g}\left(\phi_{1}\left(Y_{1}\right)+\phi_{2}\left(Y_{2}\right)\right)
$$

e os limites são 'sharp'.

Prova. A prova é direta, de maneira similar a da Proposição 1.

Lema 1. A Proposição 2 é válida para as seguintes funções:

- $\psi\left(x_{1}, x_{2}\right)=\left(x_{1}-k\right)^{+}+\left(x_{2}-k\right)^{+}, k>0$

- $\psi\left(x_{1}, x_{2}\right)=\left(x_{1}+x_{2}-k\right)^{+}, k>0$.

Prova. No primeiro caso, a prova é óbvia. Para $\left(x_{1}+x_{2}-k\right)^{+}$, devemos substituir $\phi_{i}\left(X_{i}\right)=$ $X_{i}-\frac{k}{2}, i=1,2$. Então, $\phi_{1}\left(X_{1}\right)+\phi_{2}\left(X_{2}\right) \leq_{s l} \phi_{1}\left(Y_{1}\right)+\phi_{2}\left(Y_{2}\right)$. Como consequência, e.g. Müller e Stoyan (2002), para todo $t \in \Re$

$$
\int_{t}^{\infty} P\left(X_{1}+X_{2}-k>t\right) d t \leq \int_{t}^{\infty} P\left(Y_{1}+Y_{2}-k>t\right) d t .
$$

Agora, devemos checar se

$$
\int_{t}^{\infty} P\left\{\left(X_{1}+X_{2}-k\right)^{+}>t\right\} d t \leq \int_{t}^{\infty} P\left\{\left(Y_{1}+Y_{2}-k\right)^{+}>t\right\} d t .
$$

Para $t<0$ a desigualdade acima é óbvia. Para $t \geq 0$, tem-se

$$
P\left\{\left(X_{1}+X_{2}-k\right)^{+}>t\right\}=P\left(X_{1}+X_{2}-k>t\right)
$$

$\mathrm{e}$

$$
P\left\{\left(Y_{1}+Y_{2}-k\right)^{+}>t\right\}=P\left(Y_{1}+Y_{2}-k>t\right) .
$$

Finalmente, aplicando-se (1.12), concluimos $\left(x_{1}+x_{2}-k\right)^{+} \leq_{s l}\left(y_{1}+y_{2}-k\right)^{+}$. 
Note que o cálculo dos limites (1.11) é um problema meramente computacional, ja que sabemos, por hipótese, as distribuições de $\mathbf{Y}$ e $\mathbf{Z}$. A hipótese de conhecimento parcial da cópula C, i.e., a informação sobre $C_{0}$ e $C_{1}$ entretanto, pode não ser realista.

A seguir, discutiremos duas formas extremas de dependência para obter limites não informativos.

Para alguma variável uniforme $\mathrm{U}$ em $[0,1]$, defina o seguinte vetor aleatório

$$
\left(Y_{1}, Y_{2}\right)=\left(F_{X_{1}}^{-1}(U), F_{X_{2}}^{-1}(U)\right)
$$

Esse vetor tem as mesmas marginais de $\left(X_{1}, X_{2}\right)$ e é chamado de comonotônico, veja Embrechts et al. (2003).

Lema 2 (Denneberg, 1994). Se $X$ e $Y$ são variáveis aleatórias comonotônicas, então para $q \in[0,1]$

$$
F_{X}^{-1}(q)+F_{Y}^{-1}(q)=F_{X+Y}^{-1}(q)
$$

Esse Lema é útil para o cálculo de limites para o TVaR, uma vez que o mesmo é expressado facilmente em termos das inversas das distribuições envolvidas.

Defina o seguinte vetor aleatório

$$
\left(Z_{1}, Z_{2}\right)=\left(F_{X_{1}}^{-1}(U), F_{X_{2}}^{-1}(1-U)\right)
$$

o qual tem as mesmas marginais de $\left(X_{1}, X_{2}\right)$. É chamado de contramonotônico, veja Embrechts et al. (2003). Contramonotonicidade não pode ser estendida dessa forma ao caso multivariado. Infelizmente não existe um resultado análogo ao Lema 2 para variáveis contramonotônicas.

Quando nada se conhece a respeito da cópula $C$, podemos usar o limite inferior de Frechet para $C_{0}$ e o limite superior de Fréchet para $C_{1}$. Isso pode ser feito uma vez que os vetores comonotônicos e contramonotônicos satisfazem as hipóteses do Teorema 3, sendo possível, então, usarmos a Porposição 2 no caso de nenhuma informação disponivel sobre $C$. Se a função $\psi$ é a soma dos riscos, o Lema 2 nos dá um modo de simplificarmos o cálculo do limite superior. 
Exemplo 2. Suponha que $X_{1}$ e $X_{2}$ sejam dois riscos com distribuições uniformes em $[0, \theta]$ e $[0, \gamma]$ respectivamente e que $\psi\left(X_{1}, X_{2}\right)=X_{1}+X_{2}$. Então, seguem-se os seguintes limites

$$
\begin{gathered}
\gamma+\frac{\theta-\gamma}{2}(1+p) \leq \operatorname{TVaR}\left(X_{1}+X_{2}\right) \leq \frac{\gamma+\theta}{2}(1+p) \\
\gamma+\frac{\theta-\gamma}{p+1} \leq \operatorname{PT}_{p}\left(X_{1}+X_{2}\right) \leq \frac{\theta+\gamma}{p+1}
\end{gathered}
$$

$\mathrm{e}$

$$
\gamma+\frac{(\theta-\gamma)(3+p)}{6} \leq G T_{p}\left(X_{1}+X_{2}\right) \leq \frac{(\gamma+\theta)(3+p)}{6}
$$

Pondo $\theta=\gamma=1$ podemos ver que os limites do Exemplo 1, dados pela expressão (1.9) não são 'sharp'. Por exemplo, no caso do TVaR

$$
1 \leq T \operatorname{TaR} R_{p}\left(X_{1}+X_{2}\right) \leq(1+p)
$$

enquanto que em (1.9)

$$
\frac{1+p}{2} \leq T \operatorname{VaR}\left(X_{1}+X_{2}\right) \leq \frac{3+p}{2}
$$

Exemplo 3. Suponha que $X_{1}$ e $X_{2}$ tem distribuição exponencial de parâmetros $\lambda$ e $\beta$, respectivamente. Nesse caso, não se pode obter expressões analíticas para os limites inferiores (podem ser calculados numericamente). Ainda assim, é possivel calcular limites superiores.

$$
\begin{gathered}
T \operatorname{VaR}_{p}\left(X_{1}+X_{2}\right) \leq \frac{\lambda+\beta}{\lambda \beta}(1-\ln (1-p)), \\
P T_{p}\left(X_{1}+X_{2}\right) \leq \frac{\lambda+\beta}{\lambda \beta p}
\end{gathered}
$$

e

$$
G T_{p}\left(X_{1}+X_{2}\right) \leq \frac{(\lambda+\beta)(2+p)}{2 \lambda \beta}
$$




\subsubsection{O caso multidimensional}

No caso multidimensional, as situações em que podemos usar ordem estocástica são mais restritas. Como o Teorema 3 não é mais válido para $n \geq 3$, não podemos apresentar um análogo multivariado da Proposição 2. Não obstante, é ainda possível obtermos limites superiores no caso não informativo, em algumas situações. Tal resultado pode ser útil se puder ser aplicado a funções comuns de risco, mesmo porque os investidores e demais tomadores de decisão se preocupam mais com os limites superiores, dada a aversão ao risco. Dessa forma, concluiremos que apesar dos achados dessa seção não se revestirem do atributo da generalidade, eles podem ser aplicados frequentemente.

A definição de comonotonicidade no caso multivariado pode ser estendida diretamente para o caso multidimensional, i.e., os vetores $\mathbf{X}$ e $\mathbf{Y}$ são comonotônicos

$$
\mathbf{Y}=\left(Y_{1}, \ldots, Y_{n}\right)=\left(F_{X_{1}}^{-1}(U), \ldots, F_{X_{n}}^{-1}(U)\right) \text { q.c. }
$$

onde $U$ é uniforme em $[0,1]$, e a distribuição respectiva corresponde ao limite superior de Frèchet. As propriedades da comonotonicidade são similares às do caso bidimensional. Infelizmente, o conceito de a contramonotonicidade não pode ser estendido de maneira similar ao caso bidimensional, .

Precisamos do seguinte Teorema.

Teorema 6 (Kaas et al., 2003). Para um vetor n-variado de riscos $\mathbf{X}$, defina $\mathbf{Y}$ por (1.13). Então,

$$
Y_{1}+Y_{2}+\ldots+Y_{n} \geq_{s l} X_{1}+X_{2}+\ldots+X_{n}
$$

O seguinte Lema é útil, uma vez que mostra como o Teorema 6 pode ser estendido.

Lema 3. Se $\mathbf{Y}=\left(Y_{1}, Y_{2}, \ldots, Y_{n}\right)$ pode ser representado por (1.13), então:

$$
\sum_{i=1}^{n}\left(X_{i}-k\right)^{+} \leq_{s l} \sum_{i=1}^{n}\left(Y_{i}-k\right)^{+}, k>0
$$

e

$$
\left(\sum_{i=1}^{n} X_{i}-k\right)^{+} \leq_{s l}\left(\sum_{i=1}^{n} Y_{i}-k\right)^{+}, k>0
$$


Prova. Para provar (1.14), iremos mostrar que $\left(\left(Y_{1}-k\right)^{+},\left(Y_{2}-k\right)^{+}, \ldots,\left(Y_{n}-k\right)^{+}\right)$é um vetor aleatório comonotônico com a mesma distribuição de

$$
\left(\left(X_{1}-k\right)^{+},\left(X_{2}-k\right)^{+}, \ldots,\left(X_{n}-k\right)^{+}\right)
$$

Note que para um dado $1 \leq j \leq n$, temos que

$$
\left(Y_{j}-k\right)^{+}= \begin{cases}0, & \text { se } F_{X_{j}}^{-1}(U) \leq k \\ F_{X_{j}}^{-1}(U)-k, & \text { de outra forma }\end{cases}
$$

Agora, se mostrarmos que $\left(Y_{j}-k\right)^{+}=F_{\left(X_{j}-k\right)^{+}}^{-1}(U)$ q.c., será uma consequência de $(1.13)$ e do Teorema 6. Lembre-se que $F_{X_{j}}^{-1}(U)=\sup \left\{x \in \Re \mid P\left(X_{j}<x\right) \leq U\right\}$.

Assim,

$$
P\left\{X_{j} \leq F_{X_{j}}^{-1}(U)\right\} \leq U
$$

Como consequência,

$$
P\left\{\left(X_{j}-k\right)^{+} \leq\left(F_{X_{j}}^{-1}(U)-k\right)^{+}\right\} \leq U,
$$

uma vez que $k$ é constante. Da definição de inversa, segue-se:

$$
F_{j}\left(X_{j}-k\right)^{+-1}(U)=\sup \left\{x \in \Re \mid P\left\{\left(X_{j}-k\right)^{+}<x\right\} \leq U\right\} .
$$

Destarte, $\left.F_{j}\left(X_{j}-k\right)^{+-1}(U)=F_{X_{j}}^{-1}(U)-k\right)^{+}=\left(Y_{j}-k\right)^{+}$. E o resultado segue. Agora, mostraremos (1.15). Para cada $d>0$ temos

$$
\left(\left(\sum_{i=1}^{n} x_{i}-k\right)^{+}-d\right)^{+}=\left(\sum_{i=1}^{n} x_{i}-(k+d)\right)^{+} .
$$

Essa relação e a prova do Teorema 6 (e.g. Kaas et al., 2003) nos dá que os prêmios stop-loss do lado esquerdo dessa última expressão estão ordenados. Como ambos os lados de (1.15) tem a mesma 
esperança, o resultado se segue. O mesmo vale quando $d<0$. Nesse caso,

$$
\left(\left(\sum_{i=1}^{n} x_{i}-k\right)^{+}-d\right)^{+}=\left(\left(\sum_{i=1}^{n} x_{i}-k\right)^{+}-d\right) .
$$

Estamos prontos para apresentar o resultado nuclear da presente seção.

Proposição 3. Seja $Y$ um vetor comonotônico, $g$ uma função de distorção côncava e $\psi$ representada por $\sum_{i=1}^{n} Y_{i}, \sum_{i=1}^{i=n}\left(Y_{i}-k\right)^{+}$ou $\left(\sum_{i=1}^{i=n} Y_{i}-k\right)^{+}$.Então,

$$
D_{g}\left(\psi\left(X_{1}, X_{2} \ldots, X_{n}\right)\right) \leq D_{g}\left(\psi\left(Y_{1}, Y_{2}, \ldots, Y_{n}\right)\right)
$$

e a desigualdade é 'sharp'.

Prova. A prova segue do uso dos Teoremas 4 e 6 e do Lema 3.

A Proposição 3 mostra que é possível encontrarmos limites não informativos para as medidas de risco $T V a R, P T$ e $G T$ se $\psi$ é uma das três funções apresentadas. De outra forma, a metodologia da seção (1.2) deve ser empregada.

O seguinte Lema é uma extensão multivariada do Lema 2.

Lema 4 (Denneberg, 1994). Se $X_{1}, X_{2}, \ldots, X_{n}$ são vetores comonotônicos, então para $q \in[0,1]$

$$
F_{X_{1}}^{-1}(q)+F_{X_{2}}^{-1}(q)+\ldots+F_{X_{n}}^{-1}(q)=F_{X_{1}+X_{2}+\ldots+X_{n}}^{-1}(q)
$$

Os próximos dois exemplos ilustram a teoria aqui desenvolvida.

Exemplo 4. Considere um vetor $n$-dimensional $\mathbf{X}$ de riscos, tal que a distribuição de cada $X_{i}$ é uniforme em $\left[0, \theta_{i}\right]$, para $i=1,2, \ldots, n$. Então, estendendo os resultados do Exemplo 2 e aplicando o Lema 4 para o TVaR, temos. 


$$
\begin{gathered}
T V a R_{p}\left(X_{1}+\cdots+X_{n}\right) \leq \frac{\theta_{1}+\theta_{2}+\ldots+\theta_{n}}{2}(1+p), \\
P T_{p}\left(X_{1}+\cdots+X_{n}\right) \leq \frac{\theta_{1}+\theta_{2}+\ldots+\theta_{n}}{p+1}
\end{gathered}
$$

$\mathrm{e}$

$$
G T_{p}\left(X_{1}+\cdots+X_{n}\right) \leq \frac{\left(\theta_{1}+\theta_{2}+\ldots+\theta_{n}\right)(3+p)}{6}
$$

Exemplo 5. Considere o vetor $n$-dimensional $\mathbf{X}$ de riscos, tal que cada $X_{i}$ é distribuido de maneira exponencial com parâmetro $\lambda_{i}$, para $i=1,2, \ldots, n$. Estendendo os resultados do Exemplo 3, temos

$$
\begin{gathered}
T \operatorname{VaR}_{p}\left(X_{1}+\cdots+X_{n}\right) \leq \frac{\sum_{i=1}^{n} \prod_{j \neq i} \lambda_{j}}{\prod_{i} \lambda_{i}}[1-\ln (1-p)] \\
\operatorname{PT}_{p}\left(X_{1}+\cdots+X_{n}\right) \leq \frac{\sum_{i=1}^{n} \prod_{j \neq i} \lambda_{j}}{p \prod_{i} \lambda_{i}}
\end{gathered}
$$

$\mathrm{e}$

$$
G T_{p}\left(X_{1}+\cdots+X_{n}\right) \leq \frac{(2+p) \sum_{i=1}^{n} \prod_{j \neq i} \lambda_{j}}{2 \prod_{i} \lambda_{i}}
$$

\subsubsection{Aplicação aos seguros}

Suponha que $X_{1}, X_{2}, \ldots, X_{n}$ são riscos do portfolio de uma seguradora com distribuições marginais $F_{X_{1}}, F_{X_{2}}, \ldots, F_{X_{n}}$, respectivamente. Desejamos obter limites superiores para TVaR, PT, e GT quando a função $\psi$ é a soma. Aplicando-se a Proposição 3 , com a cópula comonotônica $C_{1}$ (que

expressa a distribuição dos vetores comonotônicos. É o limite superior de Fréchet), obtemos os seguintes limites

$$
\begin{gathered}
\operatorname{TVaR}_{p}\left(X_{1}+\cdots+X_{n}\right) \leq \frac{1}{1-p} \int_{p}^{1}\left[F_{X_{1}}^{-1}(q)+F_{X_{2}}^{-1}(q)+\ldots+F_{X_{m}}^{-1}(q)\right] d q \\
P T_{p}\left(X_{1}+\cdots+X_{n}\right) \leq \int_{0}^{\infty}\left[H_{C_{1}, \psi}(t)\right]^{p} d t
\end{gathered}
$$


$\mathrm{e}$

$$
G T_{p}\left(X_{1}+\cdots+X_{n}\right) \leq \int_{0}^{\infty}\left[(1+p) \bar{H}_{C_{1}, \psi}(t)-p \bar{H}_{C_{1}, \psi}(t)^{2}\right] d t
$$

onde

$$
H_{C_{1}, \psi}(t)=P\{\psi(\mathbf{Y})<t\} \text { e } \bar{H}_{C_{1}, \psi}(t)=1-H_{C_{1}, \psi}(t)
$$

com Y sendo comonotônico.

Abaixo, mostramos os limites superiores dessas medidas de risco computados para uma carteira de 5 riscos exponencialmente distribuídos com parâmetros $\lambda_{i}=\frac{1}{i}$ para $i=1,2, \ldots, 5$ e $p=0.95$. Os valores calculados são os que seguem.

- O limite superior para o $T V a R_{0.95}\left(X_{1}+\cdots+X_{5}\right)$ é 1095 , que nos dá a perda esperada incorrida quando a mesma é maior que o quantil 0.95 ;

- O limite superior para $P T_{0.95}\left(X_{1}+\cdots+X_{5}\right)$ é 288 ;

- O limite superior para $G T_{0.95}\left(X_{1}+\cdots+X_{5}\right)$ é 404 .

A diferença entre os limites calculados é que eles se referem a princípios de prêmios diferentes.

Devemos notar que os riscos considerados nada mais são que um prêmio de seguro (prêmio puro para um risco de seguro que inclui a perda líquida esperada mais o 'load charge', que inclui despesas e comissões) para riscos não negativos $X_{i}$. De acordo com Wang et al. (1997), eles têm todas as propriedades desejáveis para uma medida de risco. Como notamos anteriormente, a Transformação de Potência, PT, pode ser usada quando se deseja incorporar diretamente a aversão ao risco do segurador no modelo, aversão essa caracterizada por $p$. Quanto maior seu valor, menor a aversão. No exemplo acima, o segurador não é tão avesso ao risco. Mas se $p$ fosse 0.1 , então o limite superior seria 2740 , obviamente muito maior que 288 , calculado para $p=0.95$.

Cada uma dessas medidas são discutidas em Wang (1996). O segurador pode escolher a mais apropriada para o cenário econômico, o ambiente que o cerca, e outras decisões gerenciais. Pode optar por calcular várias delas, como forma de analisar as interpretações que cada medida sugere. A hipótese subjacente ao uso de tais medidas é a de concorrência perfeita nos mercados, o que significa que os preços não podem ser influenciados pelos agentes isoladamente. No mercado de resseguros a 
premissa da concorrência perfeita não é, via de regra, observada, ja que o mesmo é dominado por um grupo muito pequeno de agentes.

\subsection{Conclusões}

Apresentamos duas técnicas para se calcular medidas de risco associadas a funções de distorções. A primeira abordagem falha em ser 'sharp', mas goza do atributo da generalidade, sendo válida para uma classe muito mais ampla de funções $\psi$, não sendo restritas a funções de distorção côncavas. $\mathrm{O}$ segundo método apresentado, baseado em ordem estocástica, se baseia em cálculos mais simples e fornece limites 'sharp'. Por outro lado, as condições impostas pelos teoremas respectivos são mais fortes. Todavia, os resultados são muito úteis, já que abarcam várias situações correntes no mundo prático. A técnica que deve ser escolhida, dependerá, pois, da situação em mãos. Mas deve ficar claro que a abordagem baseada em ordem estocástica deve ser usada sempre que possível.

O ordenamento de riscos pode ser usado para adaptar situações reais, descritas por modelos complexos, a modelos mais simples e tratáveis, que são mais arriscados e por isso levam a decisões mais conservadoras. Há dois motivos, ao menos, para se optar por modelos mais simples. Primeiramente, a impossibilidade de se realizar todos os cálculos do modelo mais complexo. Em segundo, geralmente há falta de informação relativa a parâmetros relevantes necessários para se estudar o modelo complexo. Destarte, podemos basear nossas deciões no modelo mais arriscado que seja compatível com nossas informações limitadas.

O material desse capítulo foi apresentado na 2nd Brazilian Conference on Statistical Modelling On Insurance and Finance, 10th Congress on Insurance: Mathematics and Economics, 9th International Vilnius Conference on Probability Theory and Mathematical Statistics, veja Gonçalves et al $(2005,2006)$ e Gonçalves \& Kolev (2006). Parcialmente, os resultados foram publicados em Gonçalves et $a l(2008 \mathrm{a})$ e Gonçalves et al (2008b). 


\section{Capítulo 2}

\section{A função de dependência de Sibuya}

Os resultados obtidos no Capítulo 1 se calcaram na teoria de cópulas. Embora muito útil, as cópulas sofrem algumas críticas, e uma delas, diz respeito ao fato de que essa função desconsidera o comportamento marginal. Nessa segunda etapa do trabalho, apresentaremos e exploraremos a função de dependência de Sibuya ${ }^{1}$, que em muitos casos se mostra mais rica que as cópulas. Em resumo, apresentaremos a função de dependência de Sibuya como uma alternativa e complemento ao uso de cópulas. Várias propriedades de tal função serão apresentadas no caso bivariado, junto com duas caracterizações. Introduziremos a função de Sibuya empírica e apresentamos um teste não paramétrico para a dependência. Um exemplo com dados reais será apresentado, com o fito de mostrar a utilidade dos resultados obtidos. No fim, consideraremos a função de Sibuya multivariada.

\subsection{Algumas limitações da metodologia de cópulas}

Começaremos por apresentar uma abordagem crítica em relação às cópulas. Para tanto, não é demais relembrar alguns fatos básicos sobre essa teoria, no caso bivariado.

Seja $H(x, y)$ a função de distribuição acumulada de duas variáveis contínuas $X$ e $Y$, com distribuições marginais $F(x)$ e $G(y)$ definidas em $\Re=(-\infty, \infty)$, respectivamente. Denote as funções inversas correspondentes por $F^{-1}(u)=\inf \{x \in \Re, F(x) \geq u\}, u \in(0,1)$ e $G^{-1}(v)=\inf \{y \in$ $\Re, G(y) \geq v\}, v \in(0,1)$. Como discutimos anteriormente, o Teorema de Sklar (e.g. Sklar (1959))

\footnotetext{
${ }^{1}$ Sibuya apresenta a função de Sibuya. Aqui, estudaremos tal com função como uma função de dependência, definida nos reais
} 
afirma que existe uma única cópula

$$
C(u, v)=H\left(F^{-1}(u), G^{-1}(v)\right), \quad u, v \in(0,1)
$$

que conecta $H(x, y)$ a $F(x)$ e $G(y)$ via

$$
H(x, y)=C(F(x), G(y)), \quad x, y \in(-\infty, \infty)
$$

Destarte, a informação da distribuição conjunta $H(x, y)$ é decomposta em dois fatores: um relativo a informação albergada pelas marginais e aquela relativa à cópula $C(u, v)$, que captura a estrutura de dependência entre $X$ e $Y$. Por outro lado, para qualquer cópula $C(u, v)$ e quaisquer marginais contínuas $F(x)$ e $G(y)$, a função $C(F(x), G(y))$ é uma função de distribuição bivariada com marginais $F(x)$ e $G(y)$, dadas por $(2.1)$.

Conseqüentemente, as cópulas permitem que se modele separadamente as marginais e a estrutura de dependência de um vetor aleatório multivariado. Trata-se de uma classe de distribuições cuja estrutura de dependência é independente do comportamento marginal, como ilustra o exemplo a seguir.

Exemplo 6. Consideremos a distribuição logística simétrica bivariada de Gumbel

$$
H_{1}(x, y)=[1+\exp (-x)+\exp (-y)]^{-1}
$$

para todo $(x, y) \in \Re^{2}$, com distribuições marginais

$$
F_{1}(x)=[1+\exp (-x)]^{-1} \quad \text { e } \quad G_{1}(y)=[1+\exp (-y)]^{-1},
$$

e a distribuição assimétrica

$$
H_{2}(x, y)= \begin{cases}\frac{(x+1)[\exp (y)-1]}{x+2 \exp (y)-1}, & \text { se }(x, y) \in[-1,1] \times[0, \infty) \\ 1-\exp (-y), & \text { se }(x, y) \in(1, \infty) \times[0, \infty) \\ 0, & \text { no restante }\end{cases}
$$

com marginais

$$
F_{2}(x)=\frac{x+1}{2}, x \in[-1,1] \quad \text { e } \quad G_{2}(y)=1-\exp (-y), y \geq 0
$$


É fácil checar que

$$
H_{i}(x, y)=\frac{F_{i}(x) G_{i}(y)}{F_{i}(x)+G_{i}(y)-F_{i}(x) G_{i}(y)}, \quad i=1,2,
$$

de forma que, não é surpresa que a cópula associada a ambas distribuições seja a mesma, isto é, $C(u, v)=\frac{u v}{u+v-u v}$, veja Nelsen (2006). Esse fato é de difícil aceitação por parte dos empiricistas, uma vez que $H_{1}(x, y)$ e $H_{2}(x, y)$ tem suportes, comportamento marginal e simetria totalmente distintos. A explicação é que o impacto das marginais na distribuição conjunta fica escondido, uma vez que quando se trabalha com cópulas, a análise fica comprimida no quadrado unitário.

O comportamento geométrico das densidades marginais (crescente, decrescente, constante, unimodal, etc) tem influência na genuína estrutura de dependência descrita pela distribuição conjunta, como mostra Fernandez e Kolev (2007). Tal influência fica escondida quando se opta pelo uso de cópulas. A conclusão de tal estudo é que deve procurar-se uma nova classe de funções de dependência, que leve em conta o comportamento marginal.

\subsection{A função de Sibuya e suas propriedades}

Encontramos na literatura uma função introduzida por Sibuya (1960), com tal propriedade, definida como

$$
H(x, y)=\Lambda(F(x), G(y)) F(x) G(y)
$$

para todo $(x, y) \in \Re^{2}$.

Trata-se de uma função muito usada na análise de valores extremos, veja Resnick (1987).

Logo apresentaremos as propriedades básicas da função $\Lambda(F(x), G(y))$ e estabeleceremos novas propriedades probabilísticas. A partir da seção (2.3), reconsideraremos a função $\Lambda$ dada em (2.2), mas dependendo do par $(x, y) \in \Re^{2}$, e apresentaremos uma série de propriedades probabilísticas. Na mesma seção, introduziremos a variável aleatória de Sibuya. Depois, construiremos a distribuição empírica de tal variável e mostraremos como ela pode ser usada para testar dependência, na seção (2.4), junto com um exemplo utilizando dados reais. Em seguida, comentaremos sumariamente os resultados obtidos anteriormente. A seção (2.5) estende a FDS para três dimensões, analisando todos os casos possíveis (i.e. conhecimento de marginais e de distribuições bivariadas, ou de algumas marginais e de algumas distribuições bivariadas). Na seção seguinte, a extensão se dá em $\Re^{n}$, mas de maneira mais geral. Buscamos encontrar propriedades semelhantes as obtidas para a FDS em 
duas dimensões. Por fim, aprsentamos uma forma de modelar clusters via função de dependência de Sibuya, como um possível trabalho futuro empregando a função de Sibuya.

De acordo com o artigo original do Sibuya (1960), a função $\Lambda(F(x), G(y))$, como definida em (2.2), tem as seguintes propriedades:

- (P1) $\Lambda(F(x), G(y))=1$, se e somente se $X$ e $Y$ são variáveis aleatórias independentes;

- (P2) Se $\alpha(x)$ e $\beta(y)$ são funções invariantes para transformações monótonas não decrescentes, as $\Lambda$-funções dos pares $(F(x), G(y))$ e $(F(\alpha(x)), G(\beta(y)))$ são as mesmas;

- (P3) $\max \left\{0, \frac{G(y)+F(x)-1}{F(x) G(y)}\right\} \leq \Lambda(F(x), G(y)) \leq \min \left\{\frac{1}{F(x)}, \frac{1}{G(y)}\right\}$.

Lema 5 (Caracterização). A função $\Lambda(F(x), G(y))$ definida por (2.2), se relaciona com a função $\Lambda_{C}(u, v)$ da cópula associada $C(u, v)$, pelas relações

$$
\Lambda(F(x), G(y))=\Lambda_{C}(F(x), G(y))
$$

$e$

$$
\Lambda_{C}(u, v)=\Lambda\left(F\left(F^{-1}(u)\right), G\left(G^{-1}(v)\right)\right), \quad(u, v) \in(0,1)
$$

Prova. A prova se segue das combinações de $(2.1)$ e $(2.2)$. Se a função $\Lambda_{C}(u, v)$ da cópula $C(u, v)$ é conhecida, então

$$
\Lambda_{C}(F(x), G(y))=\frac{C(F(x), G(y))}{F(x) G(y)}=\frac{H(x, y)}{F(x) G(y)}=\Lambda(F(x), G(y)) .
$$

Analogamente, se a função $\Lambda(F(x), G(y))$ da distribuição conjunta $H(x, y)$ é de nosso conhecimento, então,

$$
\Lambda\left(F\left(F^{-1}(u)\right), G\left(G^{-1}(v)\right)\right)=\frac{H\left(F^{-1}(u), G^{-1}(v)\right)}{F\left(F^{-1}(u)\right) G\left(G^{-1}(v)\right)}=\frac{C(u, v)}{u v}=\Lambda_{C}(u, v) .
$$

Observação 1. O Lema 5 nos diz que toda cópula $C(u, v)$ tem sua própria função de Sibuya $\Lambda_{C}(u, v)$, que é dada pela razão entre a mesma e a cópula de independência $\Pi(u, v)=u v$ 
O mesmo Lema nos diz que a partir da cópula $C(u, v)$ e de duas marginais $F(x)$ e $G(y)$, podemos obter a função $\Lambda(F(x), G(y))$ associada. Reversamente, dada $\Lambda(F(x), G(y))$ e duas marginais , podese obter a relativa a cópula correspondente, através da relação $C(u, v)=\Lambda\left(F\left(F^{-1}(u)\right), G\left(G^{-1}(v)\right)\right) u v$. Por exemplo, a função de Sibuya correspondente a cópula do exemplo 6 é $\Lambda(u, v)=(u+v-u v)^{-1}$. As funções de Sibuya $\Lambda_{1}\left(F_{1}(x), G_{1}(y)\right)$ e $\Lambda_{2}\left(F_{2}(x), G_{2}(y)\right)$ não coincidem, em geral, nos pontos $(x, y) \in \Re^{2}$ pelo fato que as distribuições marginais são diferentes (veja a continuação do Exemplo 6, a seguir).

Exemplo 6 (continuação). A função $\Lambda$ correspondente a cópula do Exemplo 6 é $\Lambda_{C}(u, v)=$ $(u+v-u v)^{-1}$. Entretanto,

$$
\Lambda_{1}\left(F_{1}(x), G_{1}(y)\right)=\frac{H_{1}(x, y)}{F_{1}(x) G_{1}(y)}=\frac{1}{F_{1}(x)+G_{1}(y)-F_{1}(x) G_{1}(y)}
$$

$\mathrm{e}$

$$
\Lambda_{2}\left(F_{2}(x), G_{2}(y)\right)=\frac{H_{2}(x, y)}{F_{2}(x) G_{2}(y)}=\frac{1}{F_{2}(x)+G_{2}(y)-F_{2}(x) G_{2}(y)}
$$

não coincidem, dada as marginais e suportes diferentes.

Nesse ponto, faz-se interessante definir a função de dependência de sobrevivência $\bar{\Lambda}(\bar{F}(x), \bar{G}(y))$, pela equação

$$
\bar{\Lambda}(\bar{F}(x), \bar{G}(y))=\frac{\bar{H}(x, y)}{\bar{F}(x) \bar{G}(y)},
$$

onde $\bar{H}(x, y)$ é a função de sobrevivência dos pares $(X, Y), \bar{F}(x)=1-F(x)$ e $\bar{G}(y)=1-G(y)$.

É fácil ver que

$$
[\bar{\Lambda}(\bar{F}(x) \bar{G}(y))-1] \bar{F}(x) \bar{G}(y)=[\Lambda(F(x), G(y))-1] F(x) G(y) .
$$

Essa relação implica que

$$
\Lambda(F(x), G(y)) \leq 1 \quad \text { se e somente se } \bar{\Lambda}(\bar{F}(x), \bar{G}(y)) \leq 1 .
$$

Equivalentemente,

$$
\Lambda(F(x), G(y)) \geq 1 \quad \text { se e somente se } \bar{\Lambda}(\bar{F}(x), \bar{G}(y)) \geq 1 .
$$


De (P3) obtemos as seguintes fronteiras de Fréchet

$$
\max \left\{0, \frac{\bar{G}(y)+\bar{F}(x)-1}{\bar{F}(x) \bar{G}(y)}\right\} \leq \bar{\Lambda}(\bar{F}(x), \bar{G}(y)) \leq \min \left\{\frac{1}{\bar{F}(x)}, \frac{1}{\bar{G}(y)}\right\}
$$

Tais limites podem ser úteis ao se estudar o comportamento assintótico de $\Lambda(F(x), G(y))$ e $\bar{\Lambda}(\bar{F}(x), \bar{G}(y))$.

Corolário 1. São válidas as seguintes relações:

$$
\lim _{x, y \rightarrow \infty} \Lambda(F(x), G(y))=\lim _{x, y \rightarrow-\infty} \bar{\Lambda}(\bar{F}(x), \bar{G}(y))=1 .
$$

Por outro lado,

$$
\lim _{x, y \rightarrow-\infty} \Lambda(F(x), G(y)) \quad e \quad \lim _{x, y \rightarrow \infty} \bar{\Lambda}(\bar{F}(x), \bar{G}(y)) .
$$

pode variar de 0 a $\infty$.

Prova. Os dois primeiros limites são óbvios. Para provar a segunda afirmação, note que quando $X$ e $Y$ são contramonotônicos e $x, y \rightarrow-\infty$, então $\Lambda(F(x), G(y))$ vai a zero, por (P3). Todavia, se $X$ e $Y$ são comonotônicos e $x, y \rightarrow-\infty$, então $\Lambda(F(x), G(y))$ diverge para $\infty$. O mesmo vale para a função de dependência de sobrevivência $\bar{\Lambda}(\bar{F}(x), \bar{G}(y))$ quando $x, y \rightarrow \infty$.

Para terminar essa seção, lembremos que $H(x, y)$ é 'positive (negative) quadrant dependent', sempre que $H(x, y) \geq(\leq) F(x) G(y)$ para todo $(x, y) \in \Re^{2}$. A próxima afirmação segue de (2.2)

Corolário 2. A função $\Lambda$ tem as seguintes propriedades:

- Se $H(x, y)$ é 'negative quadrant dependent', então $\Lambda(F(x), G(y)) \leq 1$, q.c.;

- Se $H(x, y)$ é 'positive quadrant dependent', então $\Lambda(F(x), G(y)) \geq 1$, q.c. 


\subsection{A função de dependência de Sibuya}

Na verdade, o Lema 5 mostra que a função de dependência $\Lambda(F(x), G(y))$ definida no quadrado unitário, é equivalente a cópula da distribuição $H(x, y)$, na hipótese de conhecermos $F(x)$ e $G(y)$. Isso significa que em usando $\Lambda(F(x), G(y))$ para descrever a estrutura de dependência, obteríamos conclusões semelhantes àquelas obtidas com o bem conhecido uso de cópulas.

Aqui, consideraremos a função $\Lambda(F(x), G(y))$, estendendo seu domínio, do quadrado unitário para o $\Re^{2}$, isto é, adotando os argumentos $(x, y)$. Assim, definimos uma função $\Lambda(x, y)$, tal que

$$
\Lambda(x, y)=\frac{H(x, y)}{F(x) G(y)}, \quad(x, y) \in \Re^{2}
$$

e a chamaremos de função de dependência de Sibuya, (FDS). Todas as propriedades discutidas na seção 8, também valem para a FDS.

É possivel ver em $(2.3)$ que $\Lambda(x, y)$ é uma distância de escala ('scale distance') entre a independência (dada pelo produto das marginais $F(x)$ e $G(y)$ ) e a genuína estrutura de dependência em cada ponto $(x, y) \in \Re^{2}$, representada por $H(x, y)$. Na verdade, a $\operatorname{FDS} \Lambda(x, y)$ pode ser pensada como um coeficiente de proporcionalidade (homotetia): para cada ponto fixo $\left(x_{0}, y_{0}\right) \in \Re^{2}$, a quantidade $\Lambda\left(x_{0}, y_{0}\right)$ é o número que ajusta (estica/comprime) a influenência da "independência" (i.e. $F\left(x_{0}\right) G\left(y_{0}\right)$ ), com o fito de reproduzir a verdadeira estrutura de dependência atuante em $\left(x_{0}, y_{0}\right)$.

Outra olhada em (2.3) nos permite dizer que as marginais $F(x)$ e $G(y)$ dão a contribuição de $X$ e $Y$ para a distribuição conjunta, se tratados como independentes, enquanto a $\operatorname{FDS} \Lambda(x, y)$ captura a contribuição da associação entre $X$ e $Y$ na estrutura de dependência existente. Assim, concluimos que a estrutura de dependência pode ser decomposta em 3 fatores multiplicativos: a influência de $X$ (dada por $F(x)$ ), a influência de $Y$ (dada por $G(y)$ ) e a influência do efeito de interação entre $X$ e $Y$ (dada por FDS). Essa interpretação difere a FDS das cópulas e pode ajudar a descobrir efeitos locais adicionais na estrutura de dependência postulada pela abordagem de cópulas.

Vamos ilustrar a diferença calculando as FDS's $\Lambda_{1}(x, y)$ e $\Lambda_{2}(x, y)$, correspondentes a $H_{1}(x, y)$ e $H_{2}(x, y)$, consideradas no Exemplo 6 .

Exemplo 6 (continuação). Aqui, obteremos as funções de Sibuya $\left.\Lambda_{1}(x, y)\right)$ e $\Lambda_{2}(x, y)$ correspondente a $H_{1}(x, y)$ e $H_{2}(x, y)$ consideradas no Exemplo 6. Usando (2.2) obtemos a função de Sibuya explicitamente em termos de 
$(x, y) \in[-\infty, \infty]^{2}:$

$$
\Lambda_{1}(x, y)=\frac{\left[1+e^{-x}\right]\left[1+e^{-y}\right]}{1+e^{-x}+e^{-y}}=1+\frac{e^{-(x+y)}}{1+e^{-x}+e^{-y}}
$$

e

$$
\Lambda_{2}(x, y)= \begin{cases}\frac{2}{2-(1-x) e^{-y}}>1, & \text { se }(x, y) \in[-1,1] \times[0, \infty] ; \\ 1, & \text { se }(x, y) \in(1, \infty) \times[0, \infty] ; \\ 0, & \text { no restante }\end{cases}
$$

Claro está que $\Lambda_{1}(x, y)$ e $\Lambda_{2}(x, y)$ não são iguais para $(x, y) \in \Re^{2}$. Na verdade, para $(x, y) \in$ $(1, \infty) \times[0, \infty]$, a diferença

$$
\Lambda_{1}(x, y)-\Lambda_{2}(x, y)=\frac{e^{-(x+y)}}{1+e^{-x}+e^{-y}}
$$

é sempre positiva, e diferente de 0 para valores finitos de $x$ e $y$.

No exemplo acima, a figura 1 mostra as curvas de nível para $\Lambda_{1}(x, y)=\beta$, onde $\beta$ é constante, para valores fixos $\beta: 1.2,1.3,1.5$

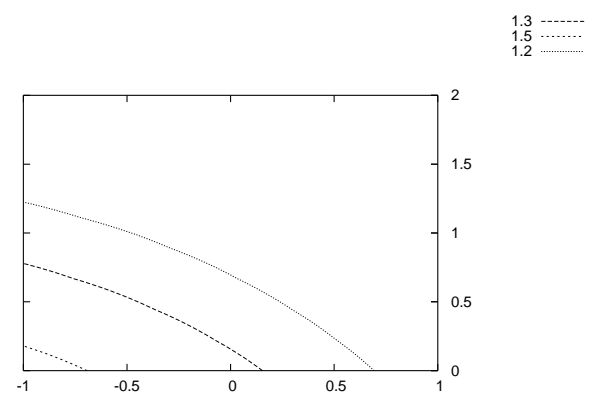

Figura 2.1: Curvas de Nível da função $\Lambda_{1}(x, y)=1.2,1.3,1.5$.

Analogamente, apresentamos o gráfico de $\Lambda_{2}(x, y)=\beta$, para os valores $\beta: 1.2,1.3,1.5$

Note que não faz sentido plotar curvas de nível quando $x \notin[-1,1]$, uma vez que nesse caso $\Lambda_{2}(x, y)$ assume apenas os valores constantes 0 e 1. Percebe-se que a função de Sibuya de ambas as distribuições se comportam de maneira diferente, embora possuam a mesma cópula.

Como conclusão, as FDS's de duas distribuições com mesma cópula em geral serão diferentes. 

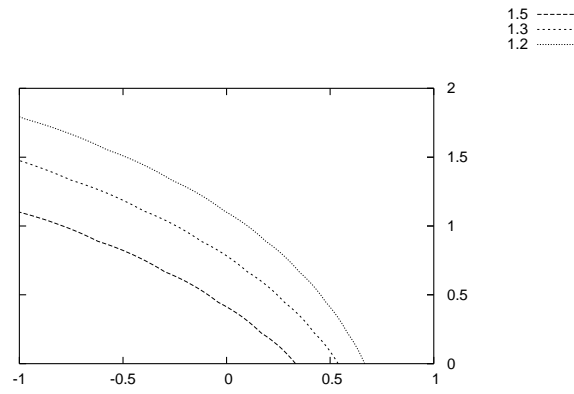

Figura 2.2: Curvas de Nível da função $\Lambda_{2}(x, y)=1.2,1.3,1.5$.

A próxima afirmação dá uma representação probabilistica da FDS.

Teorema 7. A FDS de duas variáveis aleatórias $X$ e $Y$, com as funções de distribuição correspondentes $F(x)$ e $G(y)$ podem ser representadas da forma

$$
\begin{aligned}
\Lambda(x, y) & =1+\frac{[P(Y \leq y \mid X \leq x)-P(Y \leq y \mid X>x)][1-F(x)]}{G(y)} \\
& =1+\frac{[P(X \leq x \mid Y \leq y)-P(X \leq x \mid Y>y)][1-G(y)]}{F(x)} .
\end{aligned}
$$

Prova. Levando-se em conta (2.3), temos a igualdade

$$
\Lambda(x, y)-1=\frac{P(X \leq x, Y \leq y)-P(X \leq x) P(Y \leq y)}{F(x) G(y)} .
$$

Foquemos agora no numerador e percebamos que,

$$
\begin{aligned}
P(Y \leq y) & =P(X \leq x, Y \leq y)+P(X>x, Y \leq y) \\
& =P(X \leq x, Y \leq y)+P(Y \leq y \mid X>x) P(X>x) .
\end{aligned}
$$


Multiplicando-se ambos os lados da equação por $P(X \leq x)$, temos

$$
\begin{aligned}
P(Y \leq y) P(X \leq x)= & P(X \leq x, Y \leq y) P(X \leq x) \\
& +P(Y \leq y \mid X>x) P(X>x) P(X \leq x) .
\end{aligned}
$$

No lado esquerdo da equação, substituindo-se $P(X \leq x)$ por $1-P(X>x)$, vem que

$$
\begin{aligned}
& P(X \leq x, Y \leq y)-P(X \leq x) P(Y \leq y) \\
& =P(X \leq x, Y \leq y) P(X>x)-P(Y \leq y \mid X>x) P(X>x) P(X \leq x)
\end{aligned}
$$

Fazendo a substituição no lado direito, de $P(X \leq x, Y \leq y)$ por $P(Y \leq y \mid X \leq x) P(X \leq x)$, conseguimos que

$$
\begin{aligned}
& P(X \leq x, Y \leq y)-P(X \leq x) P(Y \leq y) \\
& =[P(Y \leq y \mid X \leq x)-P(Y \leq y \mid X>x)] P(X>x) P(X \leq x)
\end{aligned}
$$

Agora, substitua tal expressão no numerador de (2.5), obtemos a primeira equação em (2.4).

A segunda representação segue analogamente.

Note que nenhuma hipótese foi feita sobre a continuidade das marginais.

Exemplo 7. Considere a família Farlie-Gumbel-Morgenstern (FGM)

$$
H(x, y)=F(x) G(y)[1+\theta(1-F(x))(1-G(y))],
$$

onde $\theta \in[-1,1]$ é um parâmetro. Usando a definição de FDS, obtemos $\Lambda(x, y)=1+\theta(1-F(x))(1-G(y))$. Agora, pelo Teorema 7

$$
\theta=\frac{P(Y \leq y \mid X \leq x)-P(Y \leq y \mid X>x)}{G(y)(1-G(y))}
$$

Como $\theta$ é uma constante e o denominador é uma função apenas de $y$, pode-se ver que a diferença $P(Y \leq y \mid X \leq x)-P(Y \leq y \mid X>x)$ deve ser independente de $x$ para a família FGM. Essa é uma característica da família FGM nunca vista antes. 
O produto $G(y)(1-G(y))$ é de no máximo $\frac{1}{4}$, assim, vale que

$$
-\frac{\theta}{4} \leq P(Y \leq y \mid X \leq x)-P(Y \leq y \mid X>x) \leq \frac{\theta}{4}
$$

para qualquer distribuição da família FGM.

Similarmente, $|P(X \leq x \mid Y \leq y)-P(X \leq x \mid Y>y)| \leq \frac{\theta}{4}$. Levando-se em conta que $\theta \in[-1,1]$, concluimos que a família FGM serve apenas para descrever dependência fraca (o que já é conhecido).

\section{Transformações monótonas da FDS}

Por questões de notação, nessa parte usaremos $\Lambda_{X, Y}(x, y)$ para enfatizar que estamos computando a FDS em relação as variáveis aleatórias $X$ e $Y$.

Primeiro, vamos discutir a propriedade de invariância (P2). Sibuya (1960) se concentrou no comportamento de $\frac{P(\alpha(X) \leq \alpha(x), \beta(Y) \leq \beta(y))}{P(\alpha(X) \leq \alpha(x)) P(\beta(Y) \leq \beta(y))}$ para funções monótonoas não decrescentes $\alpha($. and $\beta($.$) .$

Na presente seção, estamos interessados em outra transformação, a saber:

$$
\Lambda_{\alpha(X), \beta(Y)}(x, y)=\frac{P(\alpha(X) \leq x, \beta(Y) \leq y)}{P(\alpha(X) \leq x) P(\beta(Y) \leq y)}
$$

para funções monótonas $\alpha(x)$ e $\beta(y)$.

Teorema 8. Seja $\Theta_{F}(x)=\frac{F(x)}{\bar{F}(x)}$ e $\Theta_{G}(x)=\frac{G(x)}{\bar{G}(x)}$. Dadas funções arbitrárias $\alpha(x)$ e $\beta(y)$ no suporte das variáveis contínuas $X$ e $Y$, respectivamente, tal que as inversas $\alpha^{-1}($.$) e \beta^{-1}($.$) existam, temos:$

(i) Se ambas $\alpha(x)$ e $\beta(y)$ são funções crescentes, então

$$
\Lambda_{\alpha(X), \beta(Y)}(x, y)=\Lambda_{X, Y}\left(\alpha^{-1}(x), \beta^{-1}(y)\right) .
$$

(ii) Se $\alpha(x)$ é decrescente e $\beta(y)$ é uma função crescente, então

$$
\Lambda_{\alpha(X), \beta(Y)}(x, y)=1+\Theta_{F}\left(\alpha^{-1}(x)\right)\left[1-\Lambda_{X, Y}\left(\alpha^{-1}(x), \beta^{-1}(y)\right)\right] .
$$


(iii) Se $\alpha(x)$ é crescente e $\beta(y)$ é uma função decrescente, então

$$
\Lambda_{\alpha(X), \beta(Y)}(x, y)=1+\Theta_{G}\left(\beta^{-1}(x)\right)\left[1-\Lambda_{X, Y}\left(\alpha^{-1}(x), \beta^{-1}(y)\right)\right] .
$$

(iv) Se ambas $\alpha(x)$ e $\beta(y)$ são funções decrescentes, então

$$
\begin{gathered}
\Lambda_{\alpha(X), \beta(Y)}(x, y)=\frac{\bar{F}\left(\alpha^{-1}(x)\right)+\bar{G}\left(\beta^{-1}(y)\right)-1}{\bar{F}\left(\alpha^{-1}(x)\right) \bar{G}\left(\beta^{-1}(y)\right)} \\
+\Theta_{F}\left(\alpha^{-1}(x)\right) \Theta_{G}\left(\beta^{-1}(y)\right) \Lambda_{X, Y}\left(\alpha^{-1}(x), \beta^{-1}(y)\right) .
\end{gathered}
$$

Prova. As seguintes relações mostram uma sequência de igualdades entre probabilidades de eventos equivalentes.

$$
\begin{gathered}
\text { (i) } \Lambda_{\alpha(X), \beta(Y)}(x, y)=\frac{P(\alpha(X) \leq x, \beta(Y) \leq y)}{P(\alpha(X) \leq x) P(\beta(Y) \leq y)} \\
=\frac{P\left(X \leq \alpha^{-1}(x), Y \leq \beta^{-1}(y)\right)}{P\left(X \leq \alpha^{-1}(x)\right) P\left(Y \leq \beta^{-1}(y)\right)}=\frac{H\left(\alpha^{-1}(x), \beta^{-1}(y)\right)}{F\left(\alpha^{-1}(x)\right) G\left(\beta^{-1}(y)\right)}=\Lambda_{X Y}\left(\alpha^{-1}(x), \beta^{-1}(y)\right) . \\
(i i) \Lambda_{\alpha(X), \beta(Y)}(x, y)=\frac{P(\alpha(X) \leq x, \beta(Y) \leq y)}{P(\alpha(X) \leq x) P(\beta(Y) \leq y)} \\
=\frac{P\left(X>\alpha^{-1}(x), Y \leq \beta^{-1}(y)\right)}{P\left(X>\alpha^{-1}(x)\right) P\left(Y \leq \beta^{-1}(y)\right)}=\frac{P\left(Y \leq \beta^{-1}(y)\right)-H\left(\alpha^{-1}(x), \beta^{-1}(y)\right)}{\left[1-F\left(\alpha^{-1}(x)\right)\right] G\left(\beta^{-1}(y)\right)} \\
\frac{G\left(\beta^{-1}(y)\right)-H\left(\alpha^{-1}(x), \beta^{-1}(y)\right)}{\left[1-F\left(\alpha^{-1}(x)\right)\right] G\left(\beta^{-1}(y)\right)}=\frac{1}{\bar{F}\left(\alpha^{-1}(x)\right)}-\frac{F\left(\alpha^{-1}(x)\right)}{\bar{F}\left(\alpha^{-1}(x)\right)} \Lambda_{X Y}\left(\alpha^{-1}(x), \beta^{-1}(y)\right)= \\
1+\Theta_{F}\left(\alpha^{-1}(x)\right)\left[1-\Lambda_{X, Y}\left(\alpha^{-1}(x), \beta^{-1}(y)\right)\right] .
\end{gathered}
$$

(iii) Por analogia ao caso (ii).

$$
\begin{gathered}
\text { (iv) } \Lambda_{\alpha(X), \beta(Y)}(x, y)=\frac{P(\alpha(X) \leq x, \beta(Y) \leq y)}{P(\alpha(X) \leq x) P(\beta(Y) \leq y)} \\
=\frac{P\left(X>\alpha^{-1}(x), Y>\beta^{-1}(y)\right)}{P\left(X>\alpha^{-1}(x)\right) P\left(Y>\beta^{-1}(y)\right)}
\end{gathered}
$$




$$
\begin{aligned}
& =\frac{1-P\left(X \leq \alpha^{-1}(x)\right)-P\left(Y \leq \beta^{-1}(y)\right)+H\left(\alpha^{-1}(x), \beta^{-1}(y)\right)}{P\left(X>\alpha^{-1}(x)\right) P\left(Y>\beta^{-1}(y)\right)} \\
& =\frac{1-F\left(\alpha^{-1}(x)\right)+1-G\left(\beta^{-1}(y)\right)+H\left(\alpha^{-1}(x), \beta^{-1}(y)\right)-1}{\bar{F}\left(\alpha^{-1}(x)\right) \bar{G}\left(\beta^{-1}(y)\right)} .
\end{aligned}
$$

Particionando a última fração em duas e multiplicando por fatores equivalentes os numeradores e denominadores da ultima, obtemos o resultado (iv).

Nota 1 (Interpretação das relações do Teorema 8).

Caso (i). As transformações $\alpha: X \rightarrow \alpha(X)$ e $\beta: Y \rightarrow \beta(Y)$ levam $\Lambda_{\alpha(X), \beta(Y)}(x, y)$ em $\Lambda_{X, Y}\left(\alpha^{-1}(x), \beta^{-1}(y)\right)$. Ou seja, a FDS de $\alpha(X)$ e $\beta(Y)$ para o par $(x, y)$ é a de $X$ e $Y$ no ponto $\left(\alpha^{-1}(x), \beta^{-1}(y)\right)$.

Caso (ii). Em tal caso, as transformações $\alpha: X \rightarrow \alpha(X)$ e $\beta: Y \rightarrow \beta(Y)$ trazem as relações entre $\Lambda_{\alpha(X), \beta(Y)}(x, y)$ e $\Lambda_{X, Y}\left(\alpha^{-1}(x), \beta^{-1}(y)\right)$ na forma dada pelo Teorema 8 via um coeficiente de translação $1+\Theta_{F}\left(\alpha^{-1}(x)\right)$ e um coeficiente de escala $-\Theta_{F}\left(\alpha^{-1}(x)\right)$, e ambos os coeficientes dependem apenas de $\alpha(x)$ e da marginal de $X$.

Caso (iii). Interpretação análoga ao do caso (ii).

Caso (iv). Aqui, $\alpha(x)$ e $\beta(y)$ conectam $\Lambda_{\alpha(X), \beta(Y)}(x, y)$ e $\Lambda_{X, Y}\left(\alpha^{-1}(x), \beta^{-1}(y)\right)$ através do coeficiente de translação $\frac{\bar{F}\left(\alpha^{-1}(x)\right)+\bar{G}\left(\beta^{-1}(y)\right)-1}{\bar{F}\left(\alpha^{-1}(x)\right) \bar{G}\left(\beta^{-1}(y)\right)}$ e o coeficiente de escala $\Theta_{F}\left(\alpha^{-1}(x)\right) \Theta_{G}\left(\beta^{-1}(y)\right)$. Os mesmos dependem de $\alpha(x) \beta(y)$ e das marginais de $X$ e $Y$.

\subsubsection{Variável aleatória de Sibuya}

Para quaisquer pares de variáveis aleatórias contínuas $X$ e $Y$, com distribuição conjunta $H(x, y)$ e marginais $F(x)$ e $G(y)$, a quantidade

$$
\Lambda(X, Y)=\frac{H(X, Y)}{F(X) G(Y)}
$$

é também uma variável aleatória, que será chamada de variável aleatória de Sibuya.

Note que $H(X, Y)$ é uma variável aleatória com distribuição de Kendall e o denominador é o produto de duas variáveis aleatórias $U$ e $V$ uniformemente distribuídas $(0,1)$. Lembremos que a distribuição de Kendall $K(t)=P\{H(X, Y) \leq t\}$ é o sumário univariado da estrutura de dependência 
entre qualquer par de variáveis aleatórias contínuas $X$ e $Y$ com distribuição $H(x, y)$, veja Genest e Boies (2003) e Nelsen at al. (2003).

O Lema 5 nos dá uma caracterização da FDS em termos da cópula associada. Aqui, desenvolveremos uma caracterização de $\Lambda(X, Y)$ em termos da soma de variáveis aleatórias. No que se segue, consideraremos variáveis aleatórias $L, E_{1}$ e $E_{2}$, tal que $e^{L}$ tem distribuição de Kendall e $E_{1}$ e $E_{2}$ têm distribuição exponencial de parâmetro unitário.

Lema 6 (Decomposiçao). Existem variáveis aleatórias $L, E_{1}$ e $E_{2}$, tal que e ${ }^{L}$ tem distribuição de Kendall e $E_{1}$ e $E_{2}$ têm distribuição exponencial de parâmetro unitário, satisfazendo a equação

$$
\ln \Lambda(X, Y)=L+E_{1}+E_{2}
$$

Prova. De acordo com (2.6),

$$
\ln \Lambda(X, Y)=\ln H(X, Y)-\ln F(X)-\ln G(Y) .
$$

Como $F(X)$ e $G(Y)$ tem distribuição uniforme em $(0,1)$, então $E_{1}=-\ln F(X)$ e $E_{2}=-\ln G(Y)$ tem distribuição exponencial de parâmetro 1. Substituindo $L=\ln H(X, Y)$, e lembrando-se que $e^{L}=H(X, Y)$ tem distribuição de Kendall, obtemos a decomposição desejada de $\Lambda(X, Y)$.

Nota 2. Se $H(x, y)$ é 'positive quadrant dependent', então $\Lambda(X, Y)>1$ q.c. de acordo com o Corolário 2 e portanto, $-\ln H(X, Y)<-\ln F(X)-\ln G(Y)$. Dessa forma, o Lema 6 mostra que a soma de variações exponenciais de parâmetro 1 positivamente correlacionadas $E_{1}$ e $E_{2}$ 'ajustam' a variável aleatória com distribuição de Kendall até a variável de Sibuya na escala logaritimica.

Conclusões semelhantes valem quando $H(x, y)$ é 'negative quadrant dependent'.

Pelo Lema 6 concluimos que é possível decompor $\ln \Lambda(X, Y)$ na soma de três variáveis aleatórias $L, E_{1}$ e $E_{2}$, tal qual definidas acima. A implicação inversa é dada pelo seguinte lema.

Lema 7. A soma $E_{1}+E_{2}+L$ é sempre o logarítmo de uma variável aleatória de Sibuya. 
Prova. Começamos por escrever $E_{1}=-\ln U$ and $E_{2}=-\ln V$, onde $U$ e $V$ são variáveis aleatórias com distribuição uniforme em $(0,1)$. Agora, de acordo com Nelsen et al. (2003), para cada função $K(t)$ satisfazendo as propriedades da distribuição de Kendall, existe uma cópula $C(u, v)$ tal que $K(t)=P(C(u, v) \leq t),(u, v) \in[0,1]^{2}$. Destarte, $L+E_{1}+E_{2}$ pode ser reescrita como

$$
\ln W-\ln U-\ln V=\ln \frac{W}{U V}=\ln \frac{C(U, V)}{U V} .
$$

De acordo com o Lema 5, a última expressão é o logarítmo da variável aleatória de Sibuya definida em (2.6).

Combinando os Lemas 6 e 7, obtemos o seguinte

Teorema 9 (Caracterização da variável aleatória de Sibuya). A variável $\Lambda(X, Y)$ definida por (2.6) é uma variável aleatória de Sibuya se e somente se seu logarítmo puder ser escrito como a soma de três variáveis aleatórias $E_{1}, E_{2}$ e $L$.

No resto da seção, encontraremos análogos de fronteiras de Fréchet para a distribuição da variável aleatória de Sibuya. Vamos definí-la como se segue:

$$
S_{H}(t)=P(\Lambda(X, Y) \leq t), \quad t \geq 0 .
$$

Por conveniência de notação, definimos $H_{L}, H_{\Pi}$ e $H_{U}$ como funções de distribuição quando $X$ e $Y$ são variáveis contramonotônicas, independentes e comonotônicas, respectivamente. Propriedades probabilísticas da variável aleatória de Sibuya são dadas no que se segue.

Proposição 4. Sob as notações acima, as seguintes relações são válidas :

$$
\begin{gathered}
(i) \quad S_{H_{L}}(t)=I_{[t \geq 0]} ; \\
(i i) \quad S_{H_{\Pi}}(t)=I_{[t \geq 1]} ; \\
(i i i) \quad S_{H_{U}}(t)= \begin{cases}0, & \text { se } 0<t<1 ; \\
1-\frac{1}{t}, & \text { se } t \geq 1 ;\end{cases} \\
\text { (iv) } S_{H_{U}}(t) \leq S_{H_{\Pi}}(t) \leq S_{H_{L}}(t) \quad \text { para } \quad t>0 ;
\end{gathered}
$$




$$
\text { (v) } \quad S_{H_{U}}(t) \leq S_{H}(t) \leq S_{H_{L}}(t) \text { para } t>0 .
$$

onde $I_{[.]}$é a função indicadora.

Prova. Quando $X$ e $Y$ são contramonotônicas, $H(X, Y)$ é 0, quase certamente, e portanto, $P(\Lambda(X, Y) \leq$ $t)=1$ para qualquer $t \geq 0$. Obviamente, se $t<0$, então $S_{H_{L}}(t)$ será 0 , de forma que $(2.7)$ é verdadeiro.

A relação (2.8) é uma consequência do fato da FDS ser 1 se e somente se $X$ e $Y$ forem independentes, de acordo com a propriedade (P1). A relação (2.9) segue da distribuição de $U^{-1}$, onde $U$ tem distribuição uniforme em $(0,1)$. Os limites superiores e inferiores em (2.10) e (2.11) seguem das relações $(2.7)$ - (2.9) e da definição de $S_{H}(t)$.

Como uma consequência de (2.9), o valor esperado de $\Lambda(X, Y)$ não é finito se $X$ e $Y$ são variáveis aleatórias comonotônicas.

Uma outra leitura de (2.11) nos dá que é válida a seguinte ordem estocástica em distribuição

$$
\Lambda_{H_{U}}(F(X), G(Y)) \leq^{d} \Lambda_{H}(F(X), G(Y)) \leq^{d} \Lambda_{H_{L}}(F(X), G(Y))
$$

Assuma que $H_{a}(x, y)$ e $H_{b}(x, y)$ tenham as mesmas marginais $F(x)$ e $G(y)$. Definamos a ordem $\angle$ tal que $H_{a} \angle H_{b}$ se for válida a relação $H_{a}(x, y) \leq H_{b}(x, y)$ para todo $(x, y) \in \Re^{2}$. É óbvio que se $H_{a} \angle H_{b}$, então, para as FDS's correspondentes $\Lambda_{a}$ e $\Lambda_{b}$ é verdade que

$$
\Lambda_{b}(F(x), G(y)) \leq \Lambda_{a}(F(x), G(y)) \quad \text { para todo } \quad(x, y) \in \Re^{2} .
$$

\subsubsection{Limites para o valor esperado da variável aleatória de Sibuya}

É importante computar o valor esperado da variável aleatória de Sibuya, $\Lambda(X, Y)$. Como veremos na próxima seção, algumas propriedades de $\Lambda(X, Y)$ serão usadas para construir um teste não paramétrico para independência, $(\Lambda=1)$, dependência positiva $(\Lambda>1)$ e dependência negativa $(\Lambda<1)$ entre $X$ e $Y$.

Como notado após a Proposição 4, a variável aleatória de Sibuya, $\Lambda(X, Y)$ não é integrável se $X$ e 
$Y$ são comonotônicas. A tarefa de computar $E[\Lambda(X, Y)]$ pode ser difícil, como consequência da estrutura de dependência existente entre $X$ e $Y$, que refletirá nas relações entre $H(X, Y), F(X)$ e $G(Y)$. Não obstante, podemos obter um limite inferior para $E[\Lambda(X, Y)]$ usando o Lema da Decomposição.

Observação 2. É óbvio que a distribuição de Kendall de $e^{L}$ no Lema da Decomposição depende de $H(X, Y)$, de tal forma que

$$
P\left(e^{L} \leq t\right)=P(H(X, Y) \leq t):=K(t), t \in(0,1) .
$$

Uma vez que $\Lambda(X, Y)$ é sempre não negativo, temos $E[\Lambda(X, Y)] \geq 0^{2}$. Mas um outro limite inferior para esta esperança será obtido no que se segue.

Teorema 10. Para variáveis aleatórias contínuas $X$ e $Y$, é válida a seguinte desigualdade

$$
E[\Lambda(X, Y)] \geq e^{\kappa+2}
$$

onde

$$
\kappa=: \int_{0}^{1} \ln t d K(t) \leq 0
$$

Prova. Usando o Lema da Decomposição, segue-se

$$
E[\ln \Lambda(X, Y)]=E(L)+E\left(E_{1}\right)+E\left(E_{2}\right) .
$$

Uma vez que $E_{1}$ e $E_{2}$ tem distribuição exponencial unitária, $E\left(E_{1}\right)=E\left(E_{2}\right)=1$. A variável $H(X, Y)=e^{L}$ tem distribuição de Kendall, e assim

$$
\kappa=E[\ln H(X, Y)]=\int_{0}^{1} \ln t d K(t)=E(L) \leq 0,
$$

com $K(t)=P\{H(X, Y) \leq t\}$, de acordo com a Observação 2 .

\footnotetext{
${ }^{2}$ esse será chamado de limite não informativo
} 
Aplicando a desigualdade de Jensen, temos

$$
\kappa+2=E[\ln \Lambda(X, Y)] \leq \ln E\left[\Lambda_{X Y}(X, Y)\right]
$$

Destarte, $E[\Lambda(X, Y)] \geq e^{\kappa+2}$.

Observação 3. Note que a variável aleatória $L$ assume apenas valores negativos, uma vez que $0 \leq H(X, Y) \leq 1$, e assim, o valor da integral $\kappa$ está bem definido. Se $\kappa$ se aproxima de $-\infty$, então o limite inferior para $E[\Lambda(X, Y)]$ é zero. Do contrário, se $0>\kappa>-\infty$, então o limite inferior obtido para $E[\Lambda(X, Y)]$ é sempre finito e não fica abaixo do valor $e^{\kappa+2}$.

A quantidade $e^{\kappa+2}$ pode ser reescrita como $A e^{\kappa}$, onde $A=e^{2}$. Distribuições de Kendall com valores $\kappa<-2$ serão importantes quando se deseja modelar dependência negativa, usando-se o Teorema 10. Note que em qualquer caso, como o limite inferior pode ser escrito como $A e^{\kappa}$, com $A$ constante, segue-se que (2.12) só depende da distribuição de $H(X, Y)$. Assim, o ocmportamento marginal não influi no limite inferior.

Apresentamos, agora, uma afirmação relacionada a informação do limite inferior.

Proposição 5. Uma condição nenessária para o limite (2.12) ser informativo, i.e. ser maior que zero é

$$
\int_{0}^{1}[K(t) / t] d t<\infty
$$

Sob a hipótese adicional que $\lim _{t \rightarrow 0} \ln t K(t)=0$ a condição acima é também suficiente.

Prova. É fácil ver em (2.12) que o limite inferior $A e^{\kappa}$ será zero se e somente se $\kappa=-\infty$, o que é equivalente a

$$
\kappa=\int_{0}^{1} \ln t d K(t)=-\infty \quad \text { or } \quad-\kappa=\int_{0}^{1}(-\ln t) d K(t)=\infty .
$$

Usando as propriedades da esperança matemática e a desigualdade $-\kappa>0$, temos

$$
-\int_{0}^{1} \ln t d K(t)=-\left.(\ln t) K(t)\right|_{0} ^{1}+\int_{0}^{1}[K(t) / t] d t=-\lim _{t \rightarrow 0}(\ln t) K(t)=0+\int_{0}^{1}[K(t) / t] d t .
$$

Destarte, uma condição necessária para se obter um limite inferior informativo será a convergência 
da última integral imprópria.

Como $\int_{0}^{1}[K(t) / t] d t<\infty$ é a condição de integrabilidade de $\ln T$ quando $\lim _{t \rightarrow 0} \ln t K(t)=0$, a desigualdade (2.12) é também condição suficiente.

Como consequência da Proposição 5, temos:

Corolário 3. Denote por $K_{\Pi}(t)$ a distribuição de Kendall de duas variáveis aleatórias independentes. Se $H(X, Y)$ é tal que sua distribuição de Kendall obedece a desigualdade $K(t) \leq K_{\Pi}(t)$ ponto a ponto, então o limite (2.12) é sempre informativo.

Prova. Por hipótese, $H(X, Y)$ é tal que

$$
K(t)=P(H(X, Y) \leq t) \leq K_{\Pi}(t)=t-t \ln t
$$

para todo $t>0$, veja Genest e Boies (2003), e $\lim _{t \rightarrow 0} \ln [t K(t)]=0$. Destarte,

$$
\int_{0}^{1} \frac{K(t)}{t} d t \leq \int_{0}^{1} \frac{(t-t \ln t)}{t} d t=1-\int_{0}^{1} \ln t d t=2<\infty .
$$

\subsection{Distribuição de Sibuya empírica}

Antes de proceder, introduziremos algumas notações. Sejam os pares $\left(\tilde{X}_{i}, \tilde{Y}_{i}\right), i=1, \ldots, n, n$ realizações independentes do vetor aleatório $(X, Y)$. Interpretamos $\left(\tilde{X}_{1}, \ldots, \tilde{X}_{n}\right)$ como $n$ realizações independentes da variável aleatória $X$. Raciocínio análogo se aplica a $\left(\tilde{Y}_{1}, \ldots, \tilde{Y}_{n}\right)$.

As funções de distribuição empíricas de $X$ e $Y$ são $F_{n}(x)=\frac{1}{n} \#\left\{j: \tilde{X}_{j} \leq x\right\}$ e $G_{n}(y)=\frac{1}{n} \#\{j$ : $\left.\tilde{Y}_{j} \leq y\right\}$, respectivamente, e

$H_{n}(x, y)=\frac{1}{n} \#\left\{j:\left[\tilde{X}_{j} \leq x\right] \cap\left[Y_{j} \leq y\right]\right\}$ é a distribuição conjunta empírica de $X$ e $Y$, de modo que a versão empírica de $\Lambda(X, Y)$ é $\Lambda_{n}(x, y)=\frac{H_{n}(x, y)}{F_{n}(x) G_{n}(y)}$.

Estamos prontos para apresentar o seguinte Lema.

Lema 8. Se $X$ e $Y$ são variáveis aleatórias independentes, então $\Lambda_{n}(x, y) \rightarrow 1$ quase certamente 
para $n \rightarrow \infty$. Além disso, $E\left(\Lambda_{n}(x, y)\right) \rightarrow 1$.

Prova. Nesse caso temos a convergência quase certa de $H_{n}(x, y)$ a $H(x, y)$, veja Genest e Boies (2003). Como as marginais $F_{n}(x)$ e $G_{n}(y)$ convergem a $F(x)$ e $G(y)$ (em razão do Teorema de Glivenko-Cantelli), temos $\Lambda_{n}(x, y) \rightarrow 1$ q.c. sob a condição de independência. A convergência da esperança se segue do Teorema da convergência dominada.

Um modo de usar o Lema 8 para detectar dependência consiste em plotar a função de distribuição cumulativa da distribuição empírica de Sibuya e checar se ela é "similar" a $S_{H_{\Pi}}(t)$ dada pela função escada unitária (2.8). Calcular esperanças também pode ajudar.

Apresentamos agora um teste não paramétrico de independência.

Teorema 11. Sejam $\left(\tilde{X}_{k}, \tilde{Y}_{k}\right), k=1,2, \ldots, n$, cópias independentes de $X$ e $Y$. Defina $\Lambda_{n}^{k}=$ $\frac{H_{n}\left(\tilde{X}_{k}, \tilde{Y}_{k}\right)}{F_{n}\left(\tilde{X}_{k}\right) G_{n}\left(\tilde{Y}_{k}\right)}$ e denote por $\tilde{\Lambda}_{n}=\frac{1}{n} \sum_{k=1}^{n} \Lambda_{n}^{k}$. Defina $s_{n}^{2}=\frac{1}{n-1} \sum_{k=1}^{n}\left(\Lambda_{n}^{k}-\tilde{\Lambda}_{n}\right)^{2}$ e $\hat{z}_{n}=\frac{\tilde{\Lambda}_{n}-1}{s_{n}} \sqrt{n}$. Então, sob a hipótese de independência,,

$$
\lim _{n \rightarrow \infty} \hat{z}_{n}=\lim _{n \rightarrow \infty} \frac{\tilde{\Lambda}_{n}-1}{s_{n}} \sqrt{n} \sim N(0,1) .
$$

Prova. A prova é uma mera aplicação do Lema 6 e do Teorema Central do Limite.

A estatística $\hat{z}_{n}$ pode ser usada para testar se $E[\Lambda(X, Y)]=1$. Por exemplo, suponha que desejamos testar a hipótese nula

$$
H_{0}: E[\Lambda(X, Y)]=1 \quad \text { versus } \quad H_{1}: E[\Lambda(X, Y)] \neq 1
$$

sob um nível de significância $\alpha$

Então podemos proceder usando um teste normal padrão. Rejeitar a hipótese nula significa rejeitar independência. Destarte, a estatística $\hat{z}_{n}$ pode ser usada para testar se podemos rejeitar a independência. Note que $E[\Lambda(X, Y)]=1$ não confirma independência, mas a sua rejeição é a favor de dependência.

Também, sob a hipótese de dependência, podemos construir um intervalo de confiança 100(1- $\alpha) \%$ 
para $E[\Lambda(X, Y)]$. Se $c_{\frac{\alpha}{2}}$ é tal que $P\left(Z \leq c_{\frac{\alpha}{2}}\right)=\frac{\alpha}{2}$ onde $Z \sim N(0,1)$, então

$$
\left[\tilde{\Lambda}_{n}-c_{\frac{\alpha}{2}} \frac{s_{\tilde{\Lambda}_{n}}}{\sqrt{n}} ; \tilde{\Lambda}_{n}+c_{\frac{\alpha}{2}} \frac{s_{\tilde{\Lambda}_{n}}}{\sqrt{n}}\right]
$$

é o intervalo de confiança desejado.

Observação 4. Quando $n$ é pequeno ( $n<30$, por exemplo), então a estatística $T=\frac{\bar{\Lambda}_{n}-1}{s_{n}} \sqrt{n}$ pode ser aproximada por uma distribuição $t$ com $n-1$ graus de liberdade, segundo a teoria padrão; veja Lehmann (1986).

\section{Um exemplo com dados reais}

Nesta seção, aplicaremos a análise de dados reais, via FDS. As variáveis são a variação percentual do produto interno bruto (PIB) versus a variação percentual, no mesmo período, do sálario pago nas indústrias de São Paulo (SA) durante os últimos 14 anos (de 1993 a 2007), como se pode ver na próxima Tabela (e.g. www.ipeadata.gov.br). É de se esperar que quanto maiores as variações no produto interno bruto, maiores as variações da massa salarial. O diagrama de dispersão na Figura 2.3 ilustra os dados obtidos.

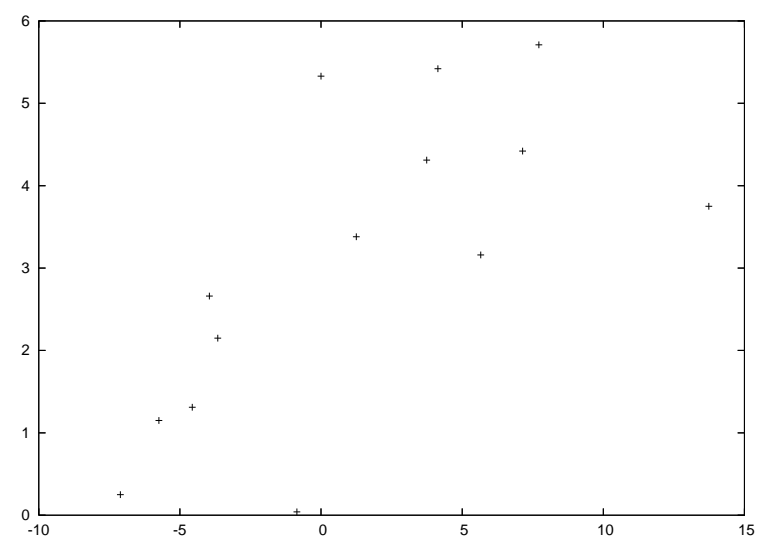

Figura 2.3: Diagrama de dispersão dos 14 pares (PIB, SA) observados.

Os valores computados da distribuição conjunta empírica $H_{n}(x, y)$ assim como os valores da FDS empírica $\Lambda_{n}(x, y)$ são apresentados na Tabela 1. 


\begin{tabular}{|c||cccc|}
\hline Ano & PIB $(x)$ & SA $(y)$ & $H_{n}(x, y)$ & $\Lambda_{n}(x, y)$ \\
\hline \hline 1994 & -0.85753 & 0.04 & 0.071429 & 2.3333 \\
1995 & -7.10875 & 0.25 & 0.071429 & 7 \\
1996 & -5.74255 & 1.15 & 0.142857 & 4.6667 \\
1997 & -4.56245 & 1.31 & 0.214285 & 3.5 \\
1998 & -3.65949 & 2.15 & 0.285714 & 2.24 \\
1999 & -3.95768 & 2.66 & 0.285714 & 2.3333 \\
\hline 2000 & 5.656018 & 3.16 & 0.5 & 1.2727 \\
2001 & 1.248078 & 3.38 & 0.5 & 1.53125 \\
2002 & 13.73966 & 3.75 & 0.642857 & 1 \\
2003 & 3.743938 & 4.31 & 0.642857 & 1.4 \\
2004 & 7.141524 & 4.42 & 0.74286 & 1.060606 \\
2005 & 0 & 5.33 & 0.5 & 1.16667 \\
2006 & 4.138104 & 5.42 & 0.74286 & 1.076923 \\
2007 & 7.715615 & 5.71 & 0.92857 & 1 \\
\hline
\end{tabular}

A Figura 2.4 apresenta os valores calculados da FDS empírica para os 14 pares observados (PIB, $\mathrm{SA})$.

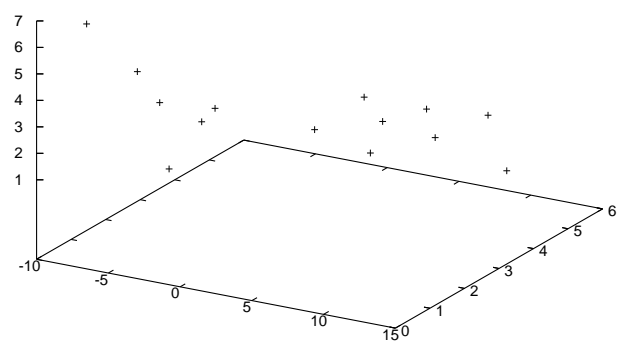

Figura 2.4: FDS empírica para os 14 pares (PIB, SA) observados. 
É interessante notar que a dependência (de acordo com a FDS calculada) é mais forte quando as observações da variável PIB são negativas. Isso significa (ao menos no período sob observação) que uma variação negativa do produto interno bruto brasileiro afeta o correspondente salário pago nas indústrias de São Paulo de maneira mais forte que o faz uma variação positiva do PIB. Na realidade, pode-se checar que nenhum valor da FDS empírica para valores positivos do PIB é maior 1.531252 (veja os últimos 8 valores computados na última coluna da Tabela 1).

Adicionalmente, o diagrama de dispersão na Figura 2.4 indica que a associação entre PIB e SA é 'positive quadrant dependent'. Tal fato não é surpresa (do ponto de vista econômico), uma vez que uma variação positiva no produto causa uma variação positiva no salário, que tende a reforçar a variação do produto e assim por diante. Raciocínio análogo vale quando a variação do produto é negativa.

Como dito, a FDS empírica mostra que o comportamento das variáveis, em relação a dependência, é distinto para valores positivos e negativos do PIB. Destarte, é interessante quebrar o conjunto de dados em dois grandes blocos: um para valores positivos do PIB e outros para valores negativos, trabalhando separadamente com cada conjunto. Além disso, no primeiro caso a distribuição a descrever os dados não deve apresentar um padrão de dependência forte, ao passo que na segunda situação, isto é, quando o PIB é negativo, devemos optar por distribuições com dependência mais forte.

A $T$-statística $T=\frac{\bar{\Lambda}_{n}-1}{s_{n}} \sqrt{n}$, tem o valor calculado de 2.71677 , e a correspondente região crítica para testar

$$
H_{0}: \text { PIB e SA são independentes contra } H_{a}: \text { PIB e SA são PQD, }
$$

com o nível de significância $\alpha=0.1$ é $[1.35, \infty)$ (baseada em uma distribuição $t$ com 13 graus de liberdade). Destarte, rejeitamos a hipótese nula de independência.

Se dividirmos o conjunto de dados em dois subconjuntos, um composto apenas por valores positivos do PIB e outros apenas por valores negativos, os valores da estatística $T$ serão $T^{+}=2.36313$ e $T^{-}=1.750334$, respectivamente. Assim, como as regiões críticas ao nível de significância $\alpha=0.1$ são $[1.415, \infty)$ and $[1.476, \infty)$, respectivamente, nós rejeitamos $H_{0}$. Essas conclusões indicam que se quebrarmos o modelo em duas partes, podemos chegar a melhores conclusões.

O exemplo com dados reais apresentados indica como se pode usar a FDS junto com as cópulas. Sugerimos o procedimento a seguir.

- (I1) Calcular a função de dependência de Sibuya empírica; 
- (I2) Dividir os dados em níveis de dependência (no dado exemplo, dois níveis foram escolhidos: um para valores positivos do PIB e outros para valores negativos).

- (I3) Escolher uma cópula apropriada (de acordo com uma função de custo apropriada, veja possíveis escolhas em Fermanian e Scaillet (2004), por exemplo) para modelar os dados em casa nível de dependência.

O procedimento (I1)-(I3) acima é mais rico que os geralmente usados na escolha ad-hoc de uma cópula paramétrica. Algumas vezes opta-se por apresentar o diagrama de dispersão de $X$ e $Y$ para separar os dados em níveis de dependência. Entretanto, o diagrama de dispersão pode não mostrar claramente os detalhes da estrutura de dependência. Se olharmos a Figura 2.3, não é óbvio que para valores positivos do PIB as variáveis não são fortemente dependentes (e "quase independentes", rejeitando a hipótese nula).

Resumindo: sugerimos, como um primeiro passo, a utilização da FDS para descriminar a estrutura de dependência (utilizando as curvas de nível comentadas na seção passada), e então usar cópulas para modelar os dados, de acordo com os distintos subconjuntos de dados. Junto com as cópulas, a FDS pode ser uma ferramenta poderosa para modelar dependência.

\subsection{A função de Sibuya em 3 dimensões}

Sejam $X_{1}, X_{2}$ e $X_{3}$ três variáveis aleatórias contínuas definidas em $\Re=(-\infty, \infty)$, com marginais $F_{i}\left(x_{i}\right)$, para $i=1,2,3$ e distribuição acumulada $H\left(x_{1}, x_{2}, x_{3}\right)$. Denotamos por $F_{i j}\left(x_{i}, x_{j}\right)$, com $1 \leq$ $i<j \leq 3$, a distribuição bivariada conjunta de $X_{i}$ e $X_{j}$, ou seja, $F_{i j}\left(x_{i}, x_{j}\right)=P\left(X_{i} \leq x_{i}, X_{j} \leq x_{j}\right)$.

Uma questão que frequentemente surge é como obter $H\left(x_{1}, x_{2}, x_{3}\right)$ a partir das marginais $F_{i}\left(x_{i}\right)$, das $F_{i j}\left(x_{i}, x_{j}\right)$ ou do conhecimento de algumas marginais e distribuições bivariadas.

Quando se trabalha com marginais univariadas, uma forma de se obter $H\left(x_{1}, x_{2}, x_{3}\right)$ a partir das mesmas se dá através do uso de cópulas. Tal como ocorre no caso bidimensional, o problema com tal abordagem é que a influência do comportamento marginal fica escondida. Além do mais, se trabalhamos com distribuições bivariadas ao invés das marginais $F_{i}\left(x_{i}\right)$, o uso de cópulas se torna problemático, veja Joe (1997) por exemplo.

Aqui, para contornar os problemas supramencionados, buscaremos expandir a função de Sibuya para o caso tri-dimensional. Consideraremos 4 possíveis casos, a saber: 
- caso 1. Conhecimento de $F_{1}\left(x_{1}\right), F_{2}\left(x_{2}\right)$ e $F_{3}\left(x_{3}\right)$.

- caso 2. Conhecimento de $F_{12}\left(x_{1}, x_{2}\right)$ e $F_{3}\left(x_{3}\right)$

- caso 3. Conhecimento de $F_{12}\left(x_{1}, x_{2}\right)$ e $F_{13}\left(x_{1}, x_{3}\right)$.

- caso 4. Conhecimento de $F_{12}\left(x_{1}, x_{2}\right), F_{13}\left(x_{1}, x_{3}\right)$ e $F_{23}\left(x_{2}, x_{3}\right)$.

Pode-se ver que, sem perda de generalidade, esses quatro casos englobam todas as situações possíveis de combinação entre as $F_{i}\left(x_{i}\right)$ e as $F_{i j}\left(x_{i}, x_{j}\right)$.

Para duas variáveis aleatórias $X_{1}$ e $X_{2}$ em $\Re^{2}$, com marginais $F_{1}\left(x_{1}\right)$ e $F_{2}\left(x_{2}\right)$ e distribuição conjunta $F_{12}\left(x_{1}, x_{2}\right)$ a função de dependência de Sibuya é definida como

$$
F_{12}\left(x_{1}, x_{2}\right)=\Lambda_{1,2}\left(F_{1}\left(x_{1}\right), F_{2}\left(x_{2}\right)\right) F_{1}\left(x_{1}\right) F_{2}\left(x_{2}\right)
$$

Aqui, explicitamos o índice 1,2 em $\Lambda_{1,2}\left(F_{1}\left(x_{1}\right), F_{2}\left(x_{2}\right)\right)$ para deixar claro que é a FS bidimensional computada para $X_{1}$ e $X_{2}$. Nosso objetivo é fazer uma expansão nos próximos quatro casos, que apresentem propriedades semelhantes à (P1) e (P3) expostas originalmente por Sibuya e constantes da seção $(2.2)$.

Embora utilizemos como argumentos da FDS estendida as marginais univariadas e as distribuições bivariadas, para acompanhar a notação original de Sibuya (1960), pode-se facilmente também usá-las como função dos pontos $\left(x_{1}, x_{2}, x_{3}\right) \in \Re^{3}$, tal como foi feito no caso bidimensional.

Por conveniência notacional, chamaremos de $\Lambda_{i j}$ a Função de Dependência de Sibuya Estendida - FDSE relacionada ao $i$-ésimo caso, $i=1, \ldots, 4$ e a $j$-ésima possivel extensão da FDS no $i$-ésimo caso.

Conhecimento de $F_{1}\left(x_{1}\right), F_{2}\left(x_{2}\right)$ e $F_{3}\left(x_{3}\right)$

Definiremos a FDSE como uma função das marginais $F_{1}\left(x_{1}\right), F_{2}\left(x_{2}\right)$ e $F_{3}\left(x_{3}\right)$. Analogamente a (2.2), é natural colocá-la como

$$
H\left(x_{1}, x_{2}, x_{3}\right)=\Lambda_{11}\left(F_{1}\left(x_{1}\right), F_{2}\left(x_{2}\right), F_{3}\left(x_{3}\right)\right) F_{1}\left(x_{1}\right) F_{2}\left(x_{2}\right) F_{3}\left(x_{3}\right) .
$$

Três propriedades interessantes se seguem de (2.14) e dos limites de Frechet. 
Lema 9. É válido que

i) $\Lambda_{11}\left(F_{1}\left(x_{1}\right), F_{2}\left(x_{2}\right), F_{3}\left(x_{3}\right)\right)=1$ se e somente se $X_{1}, X_{2}$ e $X_{3}$ forem mutuamente independentes,

ii)

$$
\begin{gathered}
\max \left\{0, \frac{F_{1}\left(x_{1}\right)+F_{2}\left(x_{2}\right)+F_{3}\left(x_{3}\right)-2}{F_{1}\left(x_{1}\right) F_{2}\left(x_{2}\right) F_{3}\left(x_{3}\right)}\right\} \leq \Lambda_{11}\left(F_{1}\left(x_{1}\right), F_{2}\left(x_{2}\right), F_{3}\left(x_{3}\right)\right) \leq \\
\min \left\{\frac{1}{F_{2}\left(x_{2}\right) F_{3}\left(x_{3}\right)}, \frac{1}{F_{1}\left(x_{1}\right) F_{3}\left(x_{3}\right)}, \frac{1}{F_{1}\left(x_{2}\right) F_{2}\left(x_{2}\right)}\right\} .
\end{gathered}
$$

iii) Se $H\left(x_{1}, x_{2}, x_{3}\right)$ é 'positive quadrant dependent'

(isto é, se $H\left(x_{1}, x_{2}, x_{3}\right) \geq F_{1}\left(x_{1}\right) F_{2}\left(x_{2}\right) F_{3}\left(x_{3}\right)$ para cada $\left(x_{1}, x_{2}, x_{3}\right) \in \Re^{3}$ ) então, $\Lambda_{11}\left(F_{1}\left(x_{1}\right), F_{2}\left(x_{2}\right), F_{3}\left(x_{3}\right)\right) \geq 1$ para todo $\left(x_{1}, x_{2}, x_{3}\right) \in \Re^{3}$. Por outro lado, se $H\left(x_{1}, x_{2}, x_{3}\right)$ é 'negative quadrant dependent'

(isto é, se $H\left(x_{1}, x_{2}, x_{3}\right) \leq F_{1}\left(x_{1}\right) F_{2}\left(x_{2}\right) F_{3}\left(x_{3}\right)$ para cada $\left.\left(x_{1}, x_{2}, x_{3}\right) \in \Re^{3}\right)$, então $\Lambda_{11}\left(F_{1}\left(x_{1}\right), F_{2}\left(x_{2}\right), F_{3}\left(x_{3}\right)\right) \leq 1$ para todo $\left(x_{1}, x_{2}, x_{3}\right) \in \Re^{3}$.

Agora, mostraremos que é possível obter (2.14) de (2.2)

Teorema 12. Para $i \neq j \neq k$, os seguintes limites são válidos

$$
\lim _{x_{i} \rightarrow \infty} \Lambda_{11}\left(F_{i}\left(x_{i}\right), F_{j}\left(x_{j}\right), F_{k}\left(x_{k}\right)\right)=\Lambda_{j, k}\left(F_{j}\left(x_{j}\right), F_{k}\left(x_{k}\right)\right), i=1,2,3
$$

Prova. Provaremos a relação para $i=1$, sem perda de generalidade

Sabemos que $\lim _{x_{1} \rightarrow \infty} H\left(x_{1}, x_{2}, x_{3}\right)=F_{23}\left(x_{2}, x_{3}\right)$. Além disso, $\lim _{x_{1} \rightarrow \infty} F_{1}\left(x_{1}\right)=1$. Assim, computando os limites em ambos os lados de (2.14), obteremos

$$
\lim _{x_{1} \rightarrow \infty} \Lambda_{11}\left(F_{1}\left(x_{1}\right), F_{2}\left(x_{2}\right), F_{3}\left(x_{3}\right)\right)=\frac{\lim _{x_{1} \rightarrow \infty} H\left(x_{1}, x_{2}, x_{3}\right)}{\lim _{x_{1} \rightarrow \infty} F_{1}\left(x_{1}\right) F_{2}\left(x_{2}\right) F_{3}\left(x_{3}\right)}=\frac{F_{23}\left(x_{2}, x_{3}\right)}{F_{2}\left(x_{2}\right) F_{3}\left(x_{3}\right)} .
$$

E o resultado se segue de (2.2). 
O Teorema 12 nos dá que se soubermos $\Lambda_{11}\left(F_{1}\left(x_{1}\right), F_{2}\left(x_{2}\right), F_{3}\left(x_{3}\right)\right)$ e as marginais $F_{1}\left(x_{1}\right), F_{2}\left(x_{2}\right)$ e $F_{3}\left(x_{3}\right)$, não apenas conheceremos $H\left(x_{1}, x_{2}, x_{3}\right)$, mas também todas as distribuições bivariadas. Ou, se soubermos apenas $\Lambda_{11}\left(F_{1}\left(x_{1}\right), F_{2}\left(x_{2}\right), F_{3}\left(x_{3}\right)\right)$, poderemos calcular todas as FDS's bidimensionais. Assim, a FDSE dada em (2.14) contém todas as informações existentes nas FDS's bivariadas correspondentes.

Seguindo a interpretação que foi dada para a FDS, podemos interpretar a função $\Lambda_{11}\left(F_{1}\left(x_{1}\right), F_{2}\left(x_{2}\right), F_{3}\left(x_{3}\right)\right)$ de forma análoga. Ela seria a homotetia que ajusta (estica/contrai) a influência da independência agindo em um dado ponto $\left(x_{1}, x_{2}, x_{3}\right) \in \Re^{3}$ para que possamos obter a verdadeira estrutura de dependência agindo naquele ponto. O produto das marginais nos dá a contribuição de $X_{1}, X_{2}$ e $X_{3}$ separadamente, enquanto a FDSE nos dá a contribuição da interação entre as três variáveis. Entretanto, como notado no Teorema 12, tal FDSE contém todas as informações das FDS's bidimensionais correspondentes. Assim, a interação captada por (2.14) é a interação dos pares possíveis, bem como a da trinca $X_{1}, X_{2}, X_{3}$.

Conhecimento de $F_{12}\left(x_{1}, x_{2}\right)$ e $F_{3}\left(x_{3}\right)$

Consideramos agora o caso em que a FDSE será uma função de $F_{12}\left(x_{1}, x_{2}\right)$ e $F_{3}\left(x_{3}\right)$. Uma maneira natural de definí-la é

$$
H\left(x_{1}, x_{2}, x_{3}\right)=\Lambda_{21}\left(F_{12}\left(x_{1}, x_{2}\right), F_{3}\left(x_{3}\right)\right) F_{12}\left(x_{1}, x_{2}\right) F_{3}\left(x_{3}\right) .
$$

Duas propriedades básicas são possíves de ser obtidas.

Lema 10. É válido que

i) $\Lambda_{21}\left(F_{12}\left(x_{1}, x_{2}\right), F_{3}\left(x_{3}\right)\right)=1$ se e somente se o par $\left(X_{1}, X_{2}\right)$ é independente de $X_{3}$. Em particular isso é válido se as variáveis aleatórias envolvidas forem mutuamente independentes.

ii)

$$
\max \left\{0, \frac{F_{12}\left(x_{1}, x_{2}\right)+F_{3}\left(x_{3}\right)-1}{F_{12}\left(x_{1}, x_{2}\right) F_{3}\left(x_{3}\right)}\right\} \leq \Lambda_{21}\left(F_{12}\left(x_{1}, x_{2}\right), F_{3}\left(x_{3}\right)\right) \leq \min \left\{\frac{1}{F_{12}\left(x_{1}, x_{2}\right)}, \frac{1}{F_{3}\left(x_{3}\right)}\right\} .
$$

Prova. A prova é uma consequência imediata de (2.16), dos limites para $H\left(x_{1}, x_{2}, x_{3}\right)$, para 
$F_{12}\left(x_{1}, x_{2}\right)$ e para $F_{3}\left(x_{3}\right)$ (e.g., Joe (1997)) e da independência.

Observação 5. Dizer que $\left(X_{1}, X_{2}\right)$ é independente de $X_{3}$ no Lema 10 i) não é equivalente a dizer que $X_{1}$ e $X_{2}$ são independentes de $X_{3}$.

A FDSE (2.16) pode ser interpretada de maneira análoga ao Caso 1. A diferença é que agora temos a contribuição de $X_{3}$ isoladamente, dada por $F_{3}\left(x_{3}\right)$ e a contribuição do par $\left(X_{1}, X_{2}\right)$ dada por $F_{12}\left(x_{1}, x_{2}\right)$, ao passo que a contribuição das três variáveis em conjunto seria dada por $\Lambda_{21}\left(F_{12}\left(x_{1}, x_{2}\right), F_{3}\left(x_{3}\right)\right)$.

Agora, considere a expressão dada por (2.2). É possível obter $\Lambda\left(F_{1}\left(x_{1}\right), F_{3}\left(x_{3}\right)\right)$ e $\Lambda\left(F_{2}\left(x_{2}\right), F_{3}\left(x_{3}\right)\right)$ de (2.16), como mostra o próximo Teorema.

Teorema 13. É verdade que, para $i \neq j$

$$
\lim _{x_{i} \rightarrow \infty} \Lambda_{21}\left(F_{12}\left(x_{i}, x_{j}\right), F_{3}\left(x_{3}\right)\right)=\Lambda_{j, 3}\left(F_{j}\left(x_{j}\right), F_{3}\left(x_{3}\right)\right), i=1,2 .
$$

Mas,

$$
\lim _{x_{3} \rightarrow \infty} \Lambda_{21}\left(F_{12}\left(x_{1}, x_{2}\right), F_{3}\left(x_{3}\right)\right)=1 .
$$

Prova. Sabemos que

$$
\lim _{x_{1} \rightarrow \infty} H\left(x_{1}, x_{2}, x_{3}\right)=F_{23}\left(x_{2}, x_{3}\right) .
$$

Além disso,

$$
\lim _{x_{1} \rightarrow \infty} F_{12}\left(x_{1}, x_{2}\right)=F_{2}\left(x_{2}\right) .
$$

Assim, computando-se o limite $\lim _{x_{1} \rightarrow \infty}$ em ambos os lados de (2.16) obtemos

$$
F_{23}\left(x_{2}, x_{3}\right)=\lim _{x_{1} \rightarrow \infty} \Lambda_{12}\left(F_{12}\left(x_{1}, x_{2}\right), F_{3}\left(x_{3}\right)\right) F_{2}\left(x_{2}\right) F_{3}\left(x_{3}\right)
$$

Destarte,

$$
\lim _{x_{1} \rightarrow \infty} \Lambda_{21}\left(F_{12}\left(x_{1}, x_{2}\right), F_{3}\left(x_{3}\right)\right)=\frac{F_{23}\left(x_{2}, x_{3}\right)}{F_{2}\left(x_{2}\right) F_{3}\left(x_{3}\right)} .
$$

Agora, por (2.2) segue-se o resultado. Analogamente, obtemos o limite para $i=2$.

A expressão final segue-se ao computar limites em ambos os lados de (2.16) e percebendo que 
$\lim _{x_{3} \rightarrow \infty} H\left(x_{1}, x_{2}, x_{3}\right)=F_{12}\left(x_{1}, x_{2}\right)$ e que $\lim _{x_{3} \rightarrow \infty} F_{3}\left(x_{3}\right)=1$.

Observação 6. O Teorema 13 se interpreta de maneira análoga ao Teorema 12. Note que, por ambos os Teoremas, é verdade que

$$
\begin{gathered}
\lim _{x_{i} \rightarrow \infty} \Lambda_{21}\left(F_{12}\left(x_{i}, x_{j}\right), F_{3}\left(x_{3}\right)\right)=\lim _{x_{i} \rightarrow \infty} \Lambda_{11}\left(F_{i}\left(x_{i}\right), F_{j}\left(x_{j}\right), F_{3}\left(x_{3}\right)\right) i=1,2 . \\
\lim _{x_{3} \rightarrow \infty} \Lambda_{21}\left(F_{12}\left(x_{1}, x_{2}\right), F_{3}\left(x_{3}\right)\right) \neq \lim _{x_{1} \rightarrow \infty} \Lambda_{11}\left(F_{1}\left(x_{1}\right), F_{2}\left(x_{2}\right), F_{3}\left(x_{3}\right)\right) .
\end{gathered}
$$

Ou seja, o comportamento limite de ambas SDFE's em $\infty$ é o mesmo para $X_{1}$ e $X_{2}$ mas não para $X_{3}$.

Conhecimento de $F_{12}\left(x_{1}, x_{2}\right)$ e $F_{13}\left(x_{1}, x_{3}\right)$

Seja, $F_{i \mid j}\left(x_{i}, x_{j}\right)=P\left(X_{i} \leq x_{i} \mid X_{j} \leq x_{j}\right)$. Agora, vamos supor o conhecimento de $F_{12}\left(x_{1}, x_{2}\right)$ e $F_{13}\left(x_{1}, x_{3}\right)$. Uma forma natural de definir a FDSE nesse caso é através da seguinte expressão

$$
H\left(x_{1}, x_{2}, x_{3}\right)=\Lambda_{31}\left(F_{12}\left(x_{1}, x_{2}\right), F_{13}\left(x_{1}, x_{3}\right)\right) F_{12}\left(x_{1}, x_{2}\right) F_{13}\left(x_{1}, x_{3}\right) .
$$

Entretanto, pode-se ver de (2.18) que $\Lambda_{31}\left(F_{12}\left(x_{1}, x_{2}\right), F_{13}\left(x_{1}, x_{3}\right)\right)$ não é 1 se as variáveis aleatórias forem independentes. Assim, vamos usar uma versão 'normalizada' de (2.18), que é

$$
\begin{gathered}
H\left(x_{1}, x_{2}, x_{3}\right)=\Lambda_{32}\left(F_{12}\left(x_{1}, x_{2}\right), F_{13}\left(x_{1}, x_{3}\right)\right) \frac{F_{12}\left(x_{1}, x_{2}\right) F_{13}\left(x_{1}, x_{3}\right)}{F_{1}\left(x_{1}\right)}= \\
\Lambda_{32}\left(F_{2 \mid 1}\left(x_{2}, x_{1}\right), F_{13}\left(x_{1}, x_{3}\right)\right) F_{2 \mid 1}\left(x_{2}, x_{1}\right) F_{13}\left(x_{1}, x_{3}\right) .
\end{gathered}
$$

Alternativamente,

$$
H\left(x_{1}, x_{2}, x_{3}\right)=\Lambda_{32}\left(F_{12}\left(x_{1}, x_{2}\right), F_{3 \mid 1}\left(x_{3}, x_{1}\right)\right) F_{12}\left(x_{1}, x_{2}\right) F_{3 \mid 1}\left(x_{3}, x_{1}\right) .
$$

Ou seja, vamos expressar a FDSE no Caso 3 como uma função de uma probabilidade condicional. É irrelevante se vamos usar $F_{3 \mid 1}\left(x_{3}, x_{1}\right)$ ou $F_{2 \mid 1}\left(x_{2}, x_{1}\right)$

Nesse caso, são válidas as seguintes propriedades. 
Lema 11. É válido que

i) $\Lambda_{32}\left(F_{2 \mid 1}\left(x_{2}, x_{1}\right), F_{13}\left(x_{1}, x_{3}\right)\right)=1$ se e somente se $X_{1}, X_{2}$ e $X_{3}$ forem mutuamente independentes.

ii) $\max \left\{0, F_{1}\left(x_{1}\right) \int_{-\infty}^{x_{1}} \max \left\{0, F_{2 \mid 1}\left(x_{2} \mid y\right)+F_{3 \mid 1}\left(x_{3} \mid y\right)-1\right\} d F_{1}(y)\right\} \leq$ $\left.\Lambda_{32}\left(F_{2 \mid 1}\left(x_{2}, x_{1}\right), F_{13}\left(x_{1}, x_{3}\right)\right) \leq F_{1}\left(x_{1}\right) \int_{-\infty}^{x_{1}} \min \left\{F_{2 \mid 1}\left(x_{2} \mid y\right), F_{3 \mid 1}\left(x_{3} \mid y\right)\right\} d F_{1}(y)\right\}$.

A propriedade ii) é uma consequência dos limites de $H\left(x_{1}, x_{2}, x_{3}\right)$ dados em Joe (1997).

O próximo Teorma nos dá o comportamento caudal de $\Lambda_{32}\left(F_{2 \mid 1}\left(x_{2}, x_{1}\right), F_{13}\left(x_{1}, x_{3}\right)\right)$.

Teorema 14. Temos que

$$
\lim _{x_{1} \rightarrow \infty} \Lambda_{32}\left(F_{2 \mid 1}\left(x_{2}, x_{1}\right), F_{13}\left(x_{1}, x_{3}\right)\right)=\Lambda\left(F_{2}\left(x_{2}\right), F_{3}\left(x_{3}\right)\right) .
$$

Todavia,

$$
\lim _{x_{2} \rightarrow \infty} \Lambda_{32}\left(F_{2 \mid 1}\left(x_{2}, x_{1}\right), F_{13}\left(x_{1}, x_{3}\right)\right)=\lim _{x_{3} \rightarrow \infty} \Lambda_{32}\left(F_{2 \mid 1}\left(x_{2}, x_{1}\right), F_{13}\left(x_{1}, x_{3}\right)\right)=1 .
$$

Prova. A prova é análoga a do Teorema 13.

A próxima Proposição nos dá uma interpretação e decomposição muito úteis de $\Lambda_{32}\left(F_{2 \mid 1}\left(x_{2}, x_{1}\right), F_{13}\left(x_{1}, x_{3}\right)\right)$.

Proposição 6. Se $F_{12}\left(x_{1}, x_{2}\right) \neq 0$ então

$$
\Lambda_{21}\left(F_{12}\left(x_{1}, x_{2}\right), F_{3}\left(x_{3}\right)\right)=\Lambda_{1,3}\left(F_{1}\left(x_{1}\right), F_{3}\left(x_{3}\right)\right) \Lambda_{32}\left(F_{2 \mid 1}\left(x_{2}, x_{1}\right), F_{13}\left(x_{1}, x_{3}\right)\right) .
$$

Prova. Por (2.16) e (2.18), obtem-se $\Lambda_{21}\left(F_{12}\left(x_{1}, x_{2}\right), F_{3}\left(x_{3}\right)\right) F_{12}\left(x_{1}, x_{2}\right) F_{3}\left(x_{3}\right)=$ $\Lambda_{32}\left(F_{2 \mid 1}\left(x_{2}, x_{1}\right), F_{13}\left(x_{1}, x_{3}\right)\right) \frac{F_{12}\left(x_{1}, x_{2}\right) F_{13}\left(x_{1}, x_{3}\right)}{F_{1}\left(x_{1}\right)}$. E o resultado se segue.

Deve-se perceber que (2.20) nos dá uma interpretação muito útil de 
$\Lambda_{32}\left(F_{2 \mid 1}\left(x_{2}, x_{1}\right), F_{13}\left(x_{1}, x_{3}\right)\right)$. Ela completa a $\operatorname{FDS} \Lambda_{1,3}\left(F_{1}\left(x_{1}\right), F_{3}\left(x_{3}\right)\right)$ para que se obtenha $\Lambda_{21}\left(F_{12}\left(x_{1}, x_{2}\right), F_{3}\left(x_{3}\right)\right)$. Uma vez que $\Lambda_{1,3}\left(F_{1}\left(x_{1}\right), F_{3}\left(x_{3}\right)\right)$ carrega a estrutura de dependência do vetor bivariado $\left(X_{1}, X_{3}\right), \Lambda_{32}\left(F_{2 \mid 1}\left(x_{2}, x_{1}\right), F_{13}\left(x_{1}, x_{3}\right)\right)$ completa essa informação a fim de que se inclua $X_{2}$, de forma que obtemos a homotetia que ajusta a 'influência' da independência em um dado ponto $\left(x_{1}, x_{2}, x_{3}\right)$ para que tenhamos a verdadeira estrutura de dependência agindo no mesmo ponto, de acordo com a interpretação de $\Lambda_{21}\left(F_{12}\left(x_{1}, x_{2}\right), F_{3}\left(x_{3}\right)\right)$.

Conhecimento de $F_{12}\left(x_{1}, x_{2}\right), F_{13}\left(x_{1}, x_{3}\right)$ e $F_{23}\left(x_{2}, x_{3}\right)$

No último caso, vamos supor o conhecimento de todas as distribuições bivariadas: $F_{12}\left(x_{1}, x_{2}\right)$, $F_{13}\left(x_{1}, x_{3}\right)$ e $F_{23}\left(x_{2}, x_{3}\right)$. É natural definir $\Lambda_{41}\left(F_{12}\left(x_{1}, x_{2}\right), F_{13}\left(x_{1}, x_{3}\right), F_{23}\left(x_{2}, x_{3}\right)\right)$ como

$$
H\left(x_{1}, x_{2}, x_{3}\right)=\Lambda_{41}\left(F_{12}\left(x_{1}, x_{2}\right), F_{13}\left(x_{1}, x_{3}\right), F_{23}\left(x_{2}, x_{3}\right)\right) F_{12}\left(x_{1}, x_{2}\right) F_{13}\left(x_{1}, x_{3}\right) F_{23}\left(x_{2}, x_{3}\right) .
$$

Todavia, não temos a propriedade que $\Lambda_{41}\left(F_{12}\left(x_{1}, x_{2}\right), F_{13}\left(x_{1}, x_{3}\right), F_{23}\left(x_{2}, x_{3}\right)\right)=1$ se e somente se os vetores são independentes. Logo, definimos a FDSE nesse caso como

$$
\begin{gathered}
H\left(x_{1}, x_{2}, x_{3}\right)=\Lambda_{42}\left(F_{12}\left(x_{1}, x_{2}\right), F_{13}\left(x_{1}, x_{3}\right), F_{23}\left(x_{2}, x_{3}\right)\right) \frac{F_{12}\left(x_{1}, x_{2}\right) F_{13}\left(x_{1}, x_{3}\right) F_{23}\left(x_{2}, x_{3}\right)}{F_{1}\left(x_{1}\right) F_{2}\left(x_{2}\right) F_{3}\left(x_{3}\right)}= \\
\Lambda_{42}\left(F_{2 \mid 1}\left(x_{2}, x_{1}\right), F_{1 \mid 3}\left(x_{1}, x_{3}\right), F_{3 \mid 2}\left(x_{3}, x_{2}\right)\right) F_{2 \mid 1}\left(x_{2}, x_{1}\right) F_{1 \mid 3}\left(x_{1}, x_{3}\right) F_{3 \mid 2}\left(x_{3}, x_{2}\right) .
\end{gathered}
$$

Expressamos a FDSE no presente caso como uma função de probabilidades condicionais. Pode-se ver que a FDSE desse caso poderia também ser expressa em termos de $F_{1 \mid 2}\left(x_{1}, x_{2}\right), F_{3 \mid 1}\left(x_{3}, x_{1}\right)$ e $F_{2 \mid 3}\left(x_{2}, x_{3}\right)$

Definamos $a_{1}=1-F_{1}\left(x_{1}\right)-F_{2}\left(x_{2}\right)-F_{3}\left(x_{3}\right)+F_{12}\left(x_{1}, x_{2}\right)+F_{13}\left(x_{1}, x_{3}\right)+F_{23}\left(x_{2}, x_{3}\right), b_{1}=$ $F_{12}\left(x_{1}, x_{2}\right)+F_{13}\left(x_{1}, x_{3}\right)-F_{1}\left(x_{1}\right), b_{2}=F_{12}\left(x_{1}, x_{2}\right)+F_{23}\left(x_{2}, x_{3}\right)-F_{2}\left(x_{2}\right)$ e $b_{3}=F_{13}\left(x_{1}, x_{3}\right)+$ $F_{23}\left(x_{2}, x_{3}\right)-F_{3}\left(x_{3}\right)$. Então, as seguintes propriedades são válidas.

Lema 12. É válido que

i) $\Lambda_{42}\left(F_{2 \mid 1}\left(x_{2}, x_{1}\right), F_{1 \mid 3}\left(x_{1}, x_{3}\right), F_{3 \mid 2}\left(x_{3}, x_{2}\right)\right)=1$ se e somente se $X_{1}, X_{2}, X_{3}$ forem mutuamente independentes. 
ii) $\max \left\{0, \frac{1}{F_{2 \mid 1}\left(x_{2}, x_{1}\right) F_{1 \mid 3}\left(x_{1}, x_{3}\right) F_{3 \mid 2}\left(x_{3}, x_{2}\right)} \max \left\{b_{1}, b_{2}, b_{3}\right\}\right\} \leq$

$$
\begin{aligned}
& \Lambda_{42}\left(F_{2 \mid 1}\left(x_{2}, x_{1}\right), F_{1 \mid 3}\left(x_{1}, x_{3}\right), F_{3 \mid 2}\left(x_{3}, x_{2}\right)\right) \leq \\
& \frac{1}{F_{2 \mid 1}\left(x_{2}, x_{1}\right) F_{1 \mid 3}\left(x_{1}, x_{3}\right) F_{3 \mid 2}\left(x_{3}, x_{2}\right)} \min \left\{F_{12}\left(x_{1}, x_{2}\right), F_{13}\left(x_{1}, x_{3}\right), F_{23}\left(x_{2}, x_{3}\right), a_{1}\right\} .
\end{aligned}
$$

A propriedade $i$ ) segue-se dos limites da distribuição conjunta dados em Joe (1997). Vamos agora analisar o comportamento limite de $\Lambda_{42}\left(F_{2 \mid 1}\left(x_{2}, x_{1}\right), F_{1 \mid 3}\left(x_{1}, x_{3}\right), F_{3 \mid 2}\left(x_{3}, x_{2}\right)\right)$ em $\infty$.

Teorema 15. Para $i \neq j \neq k$, a seguinte relação é verdadeira.

$$
\lim _{x_{i} \rightarrow \infty} \Lambda_{42}\left(F_{j \mid i}\left(x_{j}, x_{i}\right), F_{i \mid k}\left(x_{i}, x_{k}\right), F_{k \mid j}\left(x_{k}, x_{j}\right)\right)=1, i=1,2,3
$$

Prova. A prova é análoga a do Teorema 13.

A seguinte Proposição nos dá uma decomposição de $\Lambda_{42}\left(F_{2 \mid 1}\left(x_{2}, x_{1}\right), F_{1 \mid 3}\left(x_{1}, x_{3}\right), F_{3 \mid 2}\left(x_{3}, x_{2}\right)\right)$.

Proposição 7. Se $F_{12}\left(x_{1}, x_{2}\right), F_{13}\left(x_{1} . x_{3}\right)$ forem não nulas (e como consequência também o são as marginais) então

$$
\Lambda_{32}\left(F_{2 \mid 1}\left(x_{2}, x_{1}\right), F_{13}\left(x_{1}, x_{3}\right)\right)=\Lambda_{2,3}\left(F_{2}\left(x_{2}\right), F_{3}\left(x_{3}\right)\right) \Lambda_{42}\left(F_{2 \mid 1}\left(x_{2}, x_{1}\right), F_{1 \mid 3}\left(x_{1}, x_{3}\right), F_{3 \mid 2}\left(x_{3}, x_{2}\right)\right) .
$$

Prova. A prova é análoga a da Proposição 6.

Note que $\Lambda_{42}\left(F_{2 \mid 1}\left(x_{2}, x_{1}\right), F_{1 \mid 3}\left(x_{1}, x_{3}\right), F_{3 \mid 2}\left(x_{3}, x_{2}\right)\right)$ pode ser interpretada como o número que deve ser multiplicado por $\Lambda_{2,3}\left(F_{2}\left(x_{2}\right), F_{3}\left(x_{3}\right)\right)$ a fim de se obter $\Lambda_{32}\left(F_{2 \mid 1}\left(x_{2}, x_{1}\right), F_{13}\left(x_{1}, x_{3}\right)\right)$. Assim, sabemos que essa última função pode ser decomposta em outras duas.

Note, também, que quando normalizamos a extensão da FDS, como fizemos em (2.19) e (2.21), obtemos decomposições interessantes, como (2.20) e (2.22), as quais não seriam possíveis em extensões naturais tais qual (2.18). 
Observação 7. Nos últimos dois casos, trabalhamos com probabilidades condicionais, ao invés de usarmos as conjuntas bivariadas. Isso significa que podemos usar os métodos apresentados nos casos 3 e 4 apenas com o conhecimento de probabilidades condicionais e marginais, a não ser para obtermos os limites inferiores e superiores apresentados.

\subsection{A função de Sibuya multidimensional}

Agora, vamos estudar o comportamento da FDS no caso $n$-dimensional, $n>3$. Nos focaremos somente na situação em que apenas as marginais são conhecidas, uma vez que é caso mais comum, e também porque desejamos comparar os resultados com a teoria de cópulas.

Sejam $X_{1}, \ldots, X_{n}$ um vetor n-variado contínuo com marginais $F_{i}\left(x_{i}\right), i=1,2, \ldots, n$, respectivamente, e distribuição conjunta $H\left(x_{1}, \ldots, x_{n}\right),\left(x_{1}, \ldots, x_{n}\right) \in \Re^{n}$.

Definimos a versão estendida da FDS nesse caso como

$$
H\left(x_{1}, \ldots, x_{n}\right)=\Lambda_{n}\left(F_{1}\left(x_{1}\right), \ldots, F_{n}\left(x_{n}\right)\right) F_{1}\left(x_{1}\right) \ldots F_{n}\left(x_{n}\right)
$$

Ou, simplesmente,

$$
H\left(x_{1}, \ldots, x_{n}\right)=\Lambda_{n}\left(F_{1}\left(x_{1}\right), \ldots, F_{n}\left(x_{n}\right)\right) \prod_{i=1}^{n} F_{i}\left(x_{i}\right)
$$

A seguir, estendemos os resultados obtidos no caso bidimensional para $n$ dimensões. As provas e vários comentários serão omitidos, por serem análogos aos apresentados nas seções 2.2 - 2.4.

Lema 13. As seguintes asserções são verdadeiras.

i) $\Lambda_{n}\left(F_{1}\left(x_{1}\right), \ldots, F_{n}\left(x_{n}\right)\right)=1$ se e somente se $X_{1}, \ldots, X_{n}$ forem mutuamente independentes.

ii) $\min \left\{0, \frac{F_{1}\left(x_{1}\right)+\ldots+F_{n}\left(x_{n}\right)+1-n}{\prod_{i=1}^{n} F_{i}\left(x_{i}\right)}\right\} \leq \Lambda_{n}\left(F_{1}\left(x_{1}\right), \ldots, F_{n}\left(x_{n}\right)\right) \leq \min _{k}\left\{\prod_{i \neq k}\left(F_{i}\left(x_{i}\right)\right)^{-1}\right\}$.

Observação 8. Note que, enquanto o limite superior acima é sempre uma função de Sibuya válida, em geral o inferior não é, uma vez que o correspondente limite de Frechet não é sempre uma distribuição válida para $n>2$. (veja Nelsen (2006)).

Salientemos que se $n \geq 3$, uma distribuição conjunta é $H\left(x_{1}, \ldots, x_{n}\right)$ 'positive lower (negative 
upper) orthant dependent - PLOD (NUOD), ${ }^{3}$ - se $H\left(x_{1}, \ldots, x_{n}\right) \geq(\leq) \prod_{i=1}^{n} F_{i}\left(x_{i}\right)$ (e.g. Joe, 1997). Como consequência, vale o seguinte Lema.

Lema 14. As seguintes relações são válidas:

- Se $H\left(x_{1}, \ldots, x_{n}\right)$ for PLOD, então $\Lambda_{n}\left(F_{1}\left(x_{1}\right), \ldots, F_{n}\left(x_{n}\right)\right) \geq 1$.

- Se $H\left(x_{1}, \ldots, x_{n}\right)$ for $N U O D$, então $\Lambda_{n}\left(F_{1}\left(x_{1}\right), \ldots, F_{n}\left(x_{n}\right)\right) \leq 1$.

Agora, considere as distribuições conjuntas $H^{1}\left(x_{1}, \ldots, x_{n}\right)$ e $H^{2}\left(x_{1}, \ldots, x_{n}\right)$, com mesmas marginais univariadas, de forma que $F_{i}^{1}\left(x_{i}\right)=F_{i}^{2}\left(x_{i}\right)=F_{i}\left(x_{i}\right)$ para $i=1,2, \ldots, n$. Dizemos que $H^{1}\left(x_{1}, \ldots, x_{n}\right)$ é mais PLOD que $H^{2}\left(x_{1}, \ldots, x_{n}\right)$ se $H^{1}\left(x_{1}, \ldots, x_{n}\right) \leq H^{2}\left(x_{1}, \ldots, x_{n}\right)$ para todo $\left(x_{1}, \ldots, x_{n}\right) \in \Re^{n}$ (e.g., Joe, 1997). Vamos definir $\Lambda_{n}^{j}\left(F_{1}\left(x_{1}\right), \ldots, F_{n}\left(x_{n}\right)\right)$ como a FDS $n$-dimensional correspondente a $H^{j}\left(x_{1}, \ldots, x_{n}\right)$, para $j=1,2$. Então, segue-se a seguinte Proposição:

Proposição 8. Sob as condições acima, temos

$\Lambda_{n}^{1}\left(F_{1}\left(x_{1}\right), \ldots, F_{n}\left(x_{n}\right)\right) \leq \Lambda_{n}^{2}\left(F_{1}\left(x_{1}\right), \ldots, F_{n}\left(x_{n}\right)\right)$ para todo $\left(x_{1}, \ldots, x_{n}\right) \in \Re^{n}$ se e somente se $H^{1}\left(x_{1}, \ldots, x_{n}\right)$ é mais PLOD que $H^{2}\left(x_{1}, \ldots, x_{n}\right)$.

A Proposição 8 nos diz que o gráfico de $\Lambda_{n}^{1}\left(F_{1}\left(x_{1}\right), \ldots, F_{n}\left(x_{n}\right)\right)$ sempre se situa abaixo de $\Lambda_{n}^{2}\left(F_{1}\left(x_{1}\right), \ldots, F_{n}\left(x_{n}\right)\right)$ toda vez que $H^{1}\left(x_{1}, \ldots, x_{n}\right)$ for mais PLOD que $H^{2}\left(x_{1}, \ldots, x_{n}\right)$. A implicação inversa também vale. Tal característica é muito útil em procedimentos inferenciais. Por exemplo, se o gráfico de $\Lambda_{n}\left(F_{1}\left(x_{1}\right), \ldots, F_{n}\left(x_{n}\right)\right) F_{1}\left(x_{1}\right) \ldots F_{n}\left(x_{n}\right)$ fica abaixo de 1 , isso pode significar que a distribuição conjunta a ser estimada é mais PLOD que a FDS da independência (que no caso é 1) e assim, restringimos as possíveis escolhas $H\left(x_{1}, \ldots, x_{n}\right)$.

Muitas vezes, ao invés de se trabalhar com o vetor aleatório $\mathbf{X}=\left(X_{1}, \ldots, X_{n}\right)$ em si, é preciso trabalhar-se com uma função $\alpha(\mathbf{X})=\left(\alpha_{1}\left(X_{1}\right), \ldots, \alpha_{n}\left(X_{n}\right)\right)$, onde tais funções $\alpha_{i}, i=1 \ldots, n$ satisfazem uma dada propriedade. O próximo Teorema apresenta uma relação entre a FDS de uma dada função $\alpha(\mathbf{X})$ e a de $\mathbf{X}$.

\footnotetext{
${ }^{3}$ para $n=2$ nós usamos a expressão 'Positive Quadrant Dependent' e 'Negative Quadrant Dependent', respectivamente.
} 
Teorema 16. Seja $\boldsymbol{X}=\left(X_{1}, \ldots, X_{n}\right)$ um vetor aleatório $n$-dimensional contínuo. Considere o vetor $\alpha(\boldsymbol{X})=\left(\alpha_{1}\left(X_{1}\right), \ldots, \alpha_{n}\left(X_{n}\right)\right)$, tal que cada $\alpha_{i}$ é crescente na imagem de $X_{i}$, para $i=1, \ldots, n$. Então, se denotarmos $P\left(\alpha_{i}\left(X_{i}\right) \leq x_{i}\right)$ por $G_{i}\left(x_{i}\right)$, segue-se que

$$
\Lambda_{n}\left(G_{1}\left(x_{1}\right), \ldots, G_{n}\left(x_{n}\right)\right)=\Lambda_{n}\left(F_{1}\left(\alpha_{1}^{-1}\left(x_{1}\right)\right), \ldots, F_{n}\left(\alpha_{n}^{-1}\left(x_{n}\right)\right)\right) .
$$

Agora, nos perguntamos se é possível, assim como fizemos no caso tridimensional, obter a FDS de dimensões menores a partir da FDS definida no $\Re^{n}$. O próximo Teorema diz que a resposta é afirmativa.

Teorema 17. É verdade que para $1 \leq j \leq n$

$$
\lim _{x_{j} \rightarrow \infty} \Lambda_{n}\left(F_{1}\left(x_{1}\right), \ldots, F_{n}\left(x_{n}\right)\right)=\Lambda_{n-1}\left(F_{1}\left(x_{1}\right), \ldots, F_{j-1}\left(x_{j-1}\right), F_{j+1}\left(x_{j+1}\right) \ldots, F_{n}\left(x_{n}\right)\right) .
$$

Observação 9. Calculando-se limites sucessivos no lado direito de (2.24) podemos obter FDS's de dimensões ainda menores, incluindo as bidimensionais. Como consequência, podemos dizer que $\Lambda_{n}\left(F_{1}\left(x_{1}\right), \ldots, F_{n}\left(x_{n}\right)\right)$ contém toda a informação das FDS's correspondentes com dimensão menor que $n$.

Também no caso $n$-dimensional é possível obter a FDS de uma cópula de mesma dimensão, uma vez que esta nada mais é que uma distribuição em $[0,1]^{n}$ com marginais uniformes no quadrado unitário, definidas por $F_{j}\left(x_{j}\right)=u_{j}$, onde $u_{j} \in[0,1]$. Então a $\operatorname{FDS} \Lambda_{n}^{C}\left(u_{1}, \ldots, u_{n}\right)$ associada será

$$
\Lambda_{n}^{C}\left(u_{1}, \ldots, u_{n}\right)=\frac{C\left(u_{1}, \ldots, u_{n}\right)}{\Pi\left(u_{1}, \ldots, u_{n}\right)},
$$

onde $\Pi\left(u_{1}, \ldots, u_{n}\right)$ é a cópula de independência. Assim a FDS associada a uma cópula $C$ no caso $n$-dimensional é a razão entre a mesma e a cópula de independência. Com base no que foi dito, podemos estabelecer a seguinte caracterização para a FDS em $\Re^{n}$.

Teorema 18 - (Caracterização). A FDS $\Lambda_{n}\left(F_{1}\left(x_{1}\right), \ldots, F_{n}\left(x_{n}\right)\right)$ de qualquer distribuição contínua $H\left(x_{1}, \ldots, x_{n}\right)$ com marginais $F_{i}\left(x_{i}\right), i=1, \ldots, n$, é relacionada a $F D S \Lambda^{C}\left(u_{1}, \ldots, u_{n}\right)$ da respectiva 
cópula $C\left(u_{1}, \ldots, u_{n}\right)$ pelas equações

$$
\Lambda_{n}\left(F_{1}\left(x_{1}\right), \ldots, F_{n}\left(x_{n}\right)\right)=\Lambda_{n}^{C}\left(F_{1}\left(x_{1}\right), \ldots, F_{n}\left(x_{n}\right)\right)
$$

e

$$
\Lambda_{n}^{C}\left(u_{1}, \ldots, u_{n}\right)=\Lambda_{n}\left(F_{1}\left(F_{1}^{-1}\left(u_{1}\right)\right), \ldots, F_{n}\left(F_{n}^{-1}\left(u_{n}\right)\right)\right)
$$

Por fim, vamos escrever a função de Sibuya empírica $n$-dimensional. Sejam as $n$-uplas $\left(\tilde{X}_{1}{ }^{k}, \ldots, \tilde{X}_{n}{ }^{k}\right)$ $k=1,2, \ldots, m, m$ realizações independentes do vetor $\left(X_{1}, \ldots, X_{n}\right)$. Além disso, diremos que $\left(\tilde{X}_{i}{ }^{1}, \ldots, \tilde{X}_{i}{ }^{m}\right)$ serão $m$ realizações independentes da variável aleatória $X_{i}, i=1, \ldots, m$, cujas marginais empíricas serão dadas por $F_{i}^{m}\left(x_{i}\right)=\frac{1}{m} \#\left\{k: \tilde{X}_{i}^{k} \leq x_{i}\right\}$ e $H_{m}\left(x_{1}, \ldots, x_{n}\right)=$ $=\frac{1}{m} \#\left\{k:\left[\tilde{X}_{1}^{k} \leq x_{1}\right] \cap \ldots \cap\left[X_{n}^{k} \leq x_{n}\right\}\right.$ será a distribuição empírica conjunta.

A FDS n-dimensional será definida como

$$
\Lambda_{n}{ }^{m}\left(F_{1}\left(x_{1}\right), \ldots, F_{n}\left(x_{n}\right)\right)=\frac{H_{n}\left(x_{1}, \ldots, x_{n}\right)}{\prod_{i=1}^{n} F_{i}^{m}\left(x_{i}\right)}
$$

Todos os resultados empíricos obtidos para o caso bidimensional podem ser aqui estendido de maneira direta.

Pode-se notar que quando se trabalha com dados reais, a abordagem aqui apresentada pode-se unir com o uso de cópulas, de forma a dar um entendimento mais profundo sobre a estrutura de dependência subjacente aos dados. Assim, não é necessário abandonar as cópulas. Pelo contrário: a modelagem por cópulas é uma teoria bem desenvolvida, com conhecidas e importantes aplicações em finanças, atuária, biologia, economia, etc. Entretanto, como dito na introdução, a abordagem por cópulas pode ser enganosa na representação da genuína estrutura de dependência por trás dos dados, não prestando atenção a contribuição das marginais na estrutura de dependência. A FDS em muitos casos pode superar esse problema.

Os resultados mostrados neste capítulo foram apresentados em Gonçalves \& Kolev (2007, 2008c) e Gonçalves et al (2007). 


\section{Capítulo 3}

\section{Conclusões}

\subsection{Considerações finais}

O presente trabalho se divide em duas partes. Em uma primeira, estudamos uma classe de medidas de risco, chamadas medidas de risco distorcidas. Mostramos duas abordagens para se calcular limites para as mesmas, no caso de informação incompleta sobre sua estrutura de dependência. A primeira abordagem, baseada em Embrechts et al. (2003) é mais geral, entretanto, fornece piores resultados. A segunda abordagem, calcada em ordem estocástica, dá resultados melhores - 'sharp' porém, é mais restrita. A segunda parte do trabalho baseia-se na função de dependência de Sibuya. O elo existente entre as duas partes do trabalho está no fato que as informações sobre a estrutura de dependência no cálculo dos limites das medidas de risco é em geral feita por meio de cópulas. Recentemente, apareceram várias criticas à modelagem por meio de tais funções. A função de dependência de Sibuya, foi então, apresentada como uma alternativa e um complemento ao uso de cópulas. Nesse sentido, desenvolvemos uma série de propriedades sobre a FDS no caso bidimensional. Um exemplo com dados reais foi, inclusive, apresentado. No fim, estudamos com detalhes possíveis extensões da FDS para o caso tridimensional, analisando as possibilidades de conhecimento das marginais e das distribuições bivariadas dadas em Joe (1997). Em seguida, generalizamos a FDS para o caso multivariado, buscando propriedades semelhantes ao caso bivariado.

\subsection{Trabalhos futuros}

Muito ainda se pode fazer, principalmente para a função de dependência de Sibuya. Uma série de outras propriedades podem ser buscadas, tanto no caso bidimensional quanto nos outros apresentados. 
Por exemplo, podemos estudar a função de dependência de Sibuya para clusters. Sabemos que um portfolio pode ser composto de clusters, ou seja, agrupamento de ativos dentro dos quais se conhece a estrutura de dependência, mas tal estrutura entre os clusters é desconhecida. No que se segue vamos apresentar uma forma de se usar a FDS para modelar a dependência de clusters. Antes, iniciamos com algumas notações.

Suponha que o vetor aleatório $\mathbf{X}$ seja tal que $\mathbf{X}=\left(X_{m_{1}} \ldots X_{m_{k}}\right)$ onde $X_{m_{i}}=\left(X_{1}^{i}, \ldots, X_{m_{i}}^{i}\right)$ e $\sum_{i=1}^{k} m_{i}=n$. Assuma que conheçamos a distribuição conjunta de cada $X_{m_{i}}$, que será denotada por $F_{i}$. Cada $X_{m_{i}}$ será chamado de cluster. Na presente modelagem, como foi dito anteriormente, conhecemos a distribuição dentro de cada cluster, mas não a distribuição conjunta do portfolio. Esse é um contexto comum em finanças. Por exemplo, pode-se criar um cluster com indústrias de software, outro com empresas do ramo mobiliário, um outro com petrolíferas e montar um portfolio. Como cada cluster contém um ramo específico da economia, pode-se conhecer com mais facilidade a estrutura de dependência dentro de cada um, restando como trabalho conhecer a distribuição conjunta do portfolio.

Podemos definir a FDS nesse caso como

$$
H\left(x_{1}, \ldots, x_{n}\right)=\Lambda\left(F_{1}, \ldots, F_{k}\right) \prod_{i=1}^{k} F_{k}
$$

A interpretação de (3.1) é similar a de (2.23), mas agora pensamos em termos de clusters ao invés de variáveis aleatórias per se. Essa modelagem por cluster pode ser pensada como uma generalização do caso 2 apresentado na seção (2.5), quando estudamos possíveis extensões da FDS em três dimensões. Naquela situação, teríamos um agrupamento formado por $\left(X_{1}, X_{2}\right)$ e outro por $X_{3}$.

Da definição, segue-se o seguinte Lema.

Lema 15. São verdadeiras as seguintes propriedades:

i) $\Lambda\left(F_{1}, \ldots, F_{k}\right)=1$ se e somente se $X_{m_{i}}$ 's forem mutuamente independentes.

ii) $\max \left\{0, \frac{\sum_{i=1}^{k} m_{i} F_{i}-(n-1)}{\prod_{i} F_{i}}\right\} \leq \Lambda\left(F_{1}, \ldots, F_{k}\right) \leq \min _{j}\left\{\prod_{i \neq j} F_{i}^{-1}\right\}$.

Prova. A prova é direta. Mesmo assim, alguém pode se perguntar como foi obtido o limite inferior 
em $i$ i). Lembre-se que $H\left(x_{1}, \ldots, x_{n}\right)$ é maior do que a soma das marginais menos $n-1$. Mas cada marginal em um dado cluster é maior que a distribuição conjunta correspondente. Dessa forma, para o $i$-ésimo cluster $\left(X_{1}^{i}, \ldots, X_{m_{i}}^{i}\right)$, a soma das marginais das variáveis aleatórias envolvidas será maior $m_{i} F_{i}$.

Observação 10. O Lema 15 nos dá que a FDS é um se e somente se os clusters forem independentes. Em particular, isso vale quando todas as variáveis envolvidas forem independentes. Em termos econômicos, podemos pensar a independência dos clusters como se cada ramo de negócio que usamos para criar os agrupamentos fossem sistemas fechados, não influenciáveis por nada a não ser por eles mesmos. Óbviamente, não se trata de uma hipótese realista. No máximo, pode servir como uma aproximação para tornar a modelagem mais simples.

Por fim, apresentaremos um Teorema que mostra como obter a FDS de um número menor de clusters a partir da FDS geral.

Teorema 19. Vale que

$$
\lim _{X_{m_{i}} \rightarrow \infty} \Lambda\left(F_{1}, \ldots, F_{k}\right)=\Lambda\left(F_{1}, \ldots, F_{m_{i-1}}, F_{m_{i+1}}, \ldots, F_{k}\right),
$$

onde o limite $\lim _{X_{m_{i}} \rightarrow \infty}$ é feito levando-se todos os componentes do $m_{i}$-ésimo cluster para o infinito.

Prova. A prova é análoga ao do Teorema 17, fazendo sucessivas aplicações dos limites para cada variável dentro do cluster, de acordo com a observação 9 .

Ressalte-se que a abordagem empírica do caso n-dimensional pode ser aplicada para o procedimento por cluster, sem perda de generalidade.

Um estudo mais pormenorizado da FDS nesse caso pode render resultados interessantes.

Outra tarefa promissora é estudar que tipos de formas de FDS produzem determinado tipo de estrutura de dependência. 


\section{Referências Bibliográficas}

Artzner, P., Delbean, F., Eber, J.M. and Heath, D. (1999). Coherent measures of risk. Mathematical Finance 9, 203-228.

Darkiewicz, G., Dhaene, J., Goovaerts, M. (2005). Risk measures and dependencies of risks. Brazilian Journal of Probability and Statistics 19(2), 155-178.

Denneberg, D. (1990). Premium calculation: why standard deviation should be replaced by absolute deviation. ASTIN Bulletin. 20, 181-190.

Denneberg, D. (1994). Non-Additive Measure and Integral. Kluwer Academic Publishers, Boston.

Denuit. M., Genest, M. and Marceau, E. (1999). Stochastc bounds on sums of dependent risks. Insurance: Mathematics and Economics 25, 85-104.

Embrechts, P., Hoing, A. and Juri, A. (2003). Using copulae to bound the Value-at-Risk for functions of dependent risks. Finance $\&$ Stochastics 7, 146-167.

Embrechts, P., Puccetti, G. (2005). Bounds for functions of dependent risks. Working paper, available at http://www.math.ethz.ch/ embrechts/.

Fernandez, M. and Kolev, N. (2007). Bivariate density characterization by geometry of marginals. Forthcoming in Economic Quality Control 22.

Frank, M., Nelsen, R. and Schweizer, B. (1987). Best possible bounds for the distribution of sum a problem of Kolmogorov. Probability Theory and Related Fields 74, 199-211.

Genest, C. and Boies, J. (2003). Testing dependence with Kendall plots. The American Statistician $44,275-284$.

Georges, P., Lamy, A-G., Nicolas, E., Quibel, G. and Roncalli, T. (2001). Multivariate survival modelling: a unified approach with copulas. Working paper. 
Gonçalves, M., Fabris, A. and Kolev, N. (2005). Bounds for distortion function of dependent risks via copulas. In: Proceedings of the Second Brazilian Conference in Insurance and Finance (N. Kolev and P. Morettin, eds.), 122-127.

Gonçalves, M., Kolev, N. (2006). Bounds for Quantile-Based Measures of Dependent Risks Functions. In Abstracts of Communications of 9th International Vilnius Conference on Probability Theory and Mathematical Statistics, 187.

Gonçalves, M., Kolev, N. (2007) Some Probabilistic Properties of Sibuya's Dependence Function. In Proceedings of Third Brazilian Conference On Statistical Modelling On Insurance and Finance, 292-296.

Gonçalves, M., Kolev, N. (2008a). Bounds for Quantile-Based Risk Measures of Dependent Random Variables. In Economic Quality Control, 23, 55-70.

Gonçalves, M., Kolev, N. (2008b). Bounds for Distorted Risk Measures. In Economic Quality Control, accepted.

Gonçalves, M., Kolev, N. (2008c). The Sibuya's Dependence Function in Three Dimensions. In Proceedings of The First Summer School on Copulas.

Gonçalves M., Kolev, N. and Dimitrov, B. (2007). Sibuya's Function. In: Proceedings of 11th International Congress on Insurance: Mathematics and Economics, 5.

Gonçalves, M., Kolev, N., Fabris, A. (2006). Bounds for Quantile-Based Measures of Dependent Risks' Functions. In Proceedings of 10th International Congress on Insurance: Mathematics and Economics, 60-61.

Hürlimann, W. (2002). Analytical bounds for two value-at-risk functionals. ASTIN Bulletin 32, $235-265$.

Joe, H. (1997). Multivariate Models and Multivariate Dependence Concepts. New York: Chapman \& Hall.

Jorion, P. (1998). Value at Risk. McGraw-Hill, Chicago.

Kaas, R., Goovaerts, M., Dhaene, J., Denuit, M. (2003). Modern Actuarial Risk Theory, 2nd Edition. Kluwer Academic Publishers, Boston.

Lehmann, E.L. (1986). Testing Statistical Hypotheses. New York: Wiley.

Makarov, G. (1981). Estimates for the distribution function of a sum of two random variables when the marginal distributions are fixed. Theory of Probability and its Applications 26, 803-806.

Markowitz, W. (1952). Portfolio selection. Journal of Finance 7, 77-91. 
Muller, A. and Stoyan, D. (2002). Comparison Methods for Stochastic Models and Risks. Chichester: John Wiley \& Sons.

Muller, A. (1997). Stop-loss order for portfolios of dependent risks. Insurance: Mathematics and Economics 21(3), 219-223.

Nelsen, R. (2006). An Introduction to Copulas. New York: Springer.

Nelsen, R., Quesada-Molina, J., Rodrigues-Lallena, J. and Ubeda-Flores, M. (2003). Kendall distribution functions. Statistics and Probability Letters 65, 263-268.

Patton, A. (2003). Modelling asymmetric exchange rate dependence, Working paper.

Resnick, S. (1987). Extreme Values, Regular Variation, and Point Processes. New York: SpringerVerlag.

Rockafellar, R. and Uryasev, S. (2000). Optimization of conditional value-at-risk. Journal of Risk 2, 21-41.

Rockafellar, R. and Uryasev, S. (2002). Conditional value-at-risk for general loss distributions. Journal of Banking \& Finance 26, 1443-1471.

Sibuya, M. (1960). Bivariate extreme statistics. Annals of the Institute of Statistical Mathematics $11,195-210$.

Sklar, A. (1959). Fonctions de repartition a $n$ dimensions et leurs marges. Publ. Inst. Univ. Paris 8, 229-331.

Wang, S. (1996). Premium calculation by transformingthe layer premium density. ASTIN Bulletin 26, 71-92.

Wang, S., Young, V., Panjer, H. (1997). Axiomatic Characterization of Insurance Prices. Insurance: Mathematics and Economics 21, 173-183.

Wang, S. (2000). A class of distortion operators for pricing financial and insurance risks. Journal of Risk and Insurance 67, 15-36.

Williamson, R. and Downs, T. (1990). Probabilistic arithmetic: numerical methods for calculating convolutions and dependency bounds. Journal of Approximate Reasoning 4, 89-158. 